\title{
Die Wirtschaftsverfassung von Taiwan: Die Situation am Wendepunkt
}

\author{
Von Wolfgang Lasars
}

\section{Einleitung 1}

Bei der wirtschaftsverfassungsrechtlichen Diskussion in Deutschland stehen das Verhältnis von Staat und Wirtschaft, eine rechtliche Verankerung eines Wirtschaftssystems im Grundgesetz, der Bereich der Unternehmerfreiheit und so weiter im Vordergrund. Einschlägige Rechtsvergleiche beschränken sich häufig auf Europa und die USA, die Situation in außereuropäischen Ländern wird nur gelegentlich dargestelllt. 2 Roellecke hat diesbezüglich die Frage aufgeworfen,

"... ob die westeuropäische Wirtschaftsordnung beliebig in Regionen mit anderer Tradition exportiert werden kann. Ihr Erfolg legt es nahe, die Frage zu bejahen. Der Mißerfolg der sogenannten Entwicklungshilfe legt es nahe, sie zu verneinen".3

Daran anknüpfend soll hier die Wirtschaftsverfassung Taiwans untersucht werden. Die Wirtschaftsverfassung Taiwans ist besonders interessant, weil Taiwan wegen seines bisherigen enormen Wirtschaftswachstums unter den Entwicklungsländern herausragt, weswegen es ja im westlichen Ausland als einer der "vier kleinen Drachen" anerkannt wird.

Die Beurteilungen der taiwanesischen Wirtschaftsverfassung sind in Taiwan selbst gegensätzlich. Einerseits versteht sich Taiwan, d.h. die Republik China auf Taiwan, offiziell als anti-kommunistischer Repräsentant eines freien Chinas. Verfassungsrechtlich ist dies wieder deutlich geworden, als in dem Verfassungszusatz 1991 das von der Regierung in

1 Bis auf wenige bekannte chinesische Namen von Personen, Orten und Organisationen, die in der in Deutschland allgemein bekannten Schreibweise wiedergegeben werden, wird in allen anderen Fällen der Angabe chinesicher Namen und Wörter die chinesische Umschrift Hanyu pinyin verwendet.

2 Siehe etwa Yash Vyas, The Constitutional Basis for Government Regulation of Concentration of Economic Power, Monopolies and Restrictive Trade Practices: The Indian Case, VRÜ 25 (1992), S. $37 \mathrm{ff}$.

3 So am Schluß seiner rechtshistorischen Untersuchung: Gerd Roellecke, Vom Privileg zum Eigentum - Zur Entwicklung der Wirtschaftsverfassung, Der Staat 30 (1991), S. 391. Er läßt die Frage im Ergebnis offen. 
Taipei beherrschte Gebiet als "freies Gebiet" bezeichnet worden ist. ${ }^{4}$ Andererseits wird von liberalen Stimmen in Taiwan eine grundlegende Liberalisierung der Wirtschaftsverfassung Taiwans gefordert.

Bevor auf die taiwanesische Wirtschaftsverfassung im einzelnen eingegangen wird, sind kurz die traditionellen, rechtlichen Besonderheiten einer liberalen Wirtschaftsverfassung zu skizzieren und zwar deswegen, weil sie in der taiwanesischen Reformdiskussion eine besondere Rolle spielen. 5

Was unter dem Begriff "Wirtschaftsverfassung" zu verstehen ist, ist umstritten.6 Hier soll Wirtschaftsverfassung in einem weiten, materiellen Sinne begriffen werden als der Gesamtkomplex der für die Ordnung des Wirtschaftslebens eines Staates grundlegenden Normen unabhängig davon, ob es sich um Verfassungsrecht oder einfaches Recht handelt.

Bei einer Verfassung, die prinzipiell eine marktwirtschaftliche Wirtschaftsordnung voraussetzt, liegt das Schwergewicht wirtschaftsverfassungsrechtlicher Regelungen in der Garantie der wirtschaftlichen Freiheitsrechte. Diese enthalten implizit die Entscheidung, daß die Zuständigkeit zur Wirtschaftsplanung im Grundsatz den Privatrechtssubjekten zusteht.7 Das dabei zugrundeliegende Konzept der Trennung von Staat und Wirtschaft, wobei der Staat über die Steuergesetzgebung am Ertrag der produzierenden Wirtschaft beteiligt ist, soll einerseits der Wirtschaft es ermöglichen, ihre Leistungsfähigkeit zu entfalten. Zum anderen soll die Trennung von Staat und Wirtschaft die Kontrolle der Wirtschaft durch den Staat erleichtern und damit die Wahrscheinlichkeit erhöhen, daß den allgemeinen, nichtwirtschaftlichen Interessen, den öffentlichen, nicht in Geld ausdrückbaren Zielen Rechnung getragen wird. 8

Im Einzelnen sind Berufsfreiheit und Eigentumsgarantie die beiden Säulen der wirtschaftlichen Freiheit. Die Berufsfreiheit schützt den Erwerb, die individuelle Leistung, die Eigentumsgarantie vor allem den durch eigene Arbeit und Leistung erworbenen Bestand an

4 Siehe zu diesem ersten Schritt der taiwanesischen Verfassungsreform im einzelnen: Wolfgang Lasars, "Rückkehr zur Verfassung, Reform der Verfassung oder Erlaß einer neuen Verfassung? Ein Zwischenbericht über die demokratische Reform im national-chinesischen Verfassungsrecht", VRÜ 25 (1992), S. 115-160, mit einer Übersetzung u.a. dieser Norm.

5 Kritisch $\mathrm{zu}$ dem traditionellen Verständnis von einer liberalen Wirtschaftsverfassung mit Hinweis auf die in Art. 109 GG und dem Gesetz zur Förderung der Stabilität und des Wachstums der Wirtschaft vom 8. Juni 1967 der Bundesregierung zugewiesenen Aufgaben, Heinz Wagner, Öffentlicher Haushalt und Wirtschaft, VVDStRL Heft 27 (1969), S. $47 \mathrm{ff}$.

$6 \mathrm{Zu}$ den verschiedenen Definitionen von "Wirtschaftsverfassung" siehe Winfried Veelken, Wirtschaftsverfassung im Systemvergleich, RabelsZ 55 (1991), S. 464 ff.

7 Veelken, RabelsZ 55 (1991), S. 468.

8 Klaus Vogel, Der Finanz- und Steuerstaat, in: Isensee/Kirchhof (Hrsg.), Handbuch des Staatsrechts, Band 1, Heidelberg, 1987, S. 1176 f. Rdnr. 59 f. 
vermögenswerten Gütern. ${ }^{9}$ Insbesondere in der Rechtsprechung des Bundesverfassungsgerichtes wird hierbei der Zusammenhang von Eigentum und Freiheit hervorgehoben.10

Die vorliegende Untersuchung beschränkt sich auf die Untermehmerfreiheit, um durch diese Konzentration die Einzigartigkeit der taiwanesischen Wirtschaftsverfassung und ihre wesentlichen Wirkungen auf die taiwanesische Wirtschaft herauszuarbeiten. Der verfassungsrechtlich geschützte Bereich der Unternehmerfreiheit ist weit gefächert. Die Unternehmerfreiheit umfaßt die Freiheit der Gründung eines Unternehmens, die Freiheit des Marktzutritts, die Organisationsfreiheit des Unternehmers, die Freiheit der Unternehmensführung, die Freiheit der marktmäßigen Betätigung, und hierbei insbesondere die Vertrags-, die Preis-, die Wettbewerbs-, die Vertriebs- und die Absatzfreiheit, und schließlich den Schutz des Unternehmensbestandes. 11

In der deutschen Verfassungslehre ist schließlich zutreffend hervorgehoben worden, daß "das Problem der Grundrechtsgeltung und der Effektivität der wirtschaftlichen Freiheiten ... in der Grundrechtsverwirklichung (besteht)"12. Dies berücksichtigend sollen hier die Ergebnisse der jüngsten, kritischen, wirtschaftswissenschaftlichen Forschung Taiwans vorgestellt werden.

\section{Die Rechtsgrundlagen der Unternehmerfreiheit}

Verfassungsrechtliche Grundlage der Unternehmerfreiheit ist Art. 15 der am 25. Dezember 1947 inkraftgetretenen Verfassung der Republik China (Verf), in welchem es heißt:

"Das Lebensrecht, das Arbeitsrecht und das Vermögensrecht des Volkes sind zu gewährleisten".

Die Berufsfreiheit, im Chinesischen wörtlich das "Arbeitsrecht" (Gongzuo quan), wird nach der herrschenden Verfassungslehre13 verstanden als Recht vor allem der Arbeitnehmer, weswegen es hier nicht weiter zu vertiefen ist.

Das Eigentum, wörtlich das "Vermögensrecht" (Caichan quan), wird verfassungsrechtlich garantiert, das System der privaten Eigentumsordnung wird von der Verfassung aner-

9 BVerfGE 30, 292 (334) - Mindestbevorratung.

10 BVerfGE 50, 290 ff. (339) - Mitbestimmungsgesetz.

11 Fritz Ossenbühl, Die Freiheiten des Unternehmers nach dem Grundgesetz, AöR 115 (1990), S. 12 ff.

12 Ossenbühl, AöR 115 (1990), S. 30, meine Hervorhebung.

13 Lin Jidong, Zhonghua minguo xianfa zhu tiao shiyi (Kommentar zur Verfassung der Republik China), Taipei, Band I, 6. Aufl. 1990, Art. 15, S. 243 ff. 
kannt. ${ }^{14}$ In Taiwan wie in Deutschland umfaßt dabei die verfassungsrechtliche Eigentumsgarantie in ihrem rechtlichen Gehalt sowohl die Privatnützigkeit als auch die Verfügungsbefugnis über den Eigentumsgegenstand.15 Das Eigentumsrecht wird als Freiheitsrecht gegenüber dem Staat verstanden.

In dem bereits am 30. November 1929 verkündeten Dritten Abschnitt Sachenrecht des Zivilgesetzbuches wird betreffend das Eigentum -mit fast gleichlautenden Worten wie in $\S 903$ BGB - bestimmt:

"§ 765. Der Eigentümer kann innerhalb der vom Gesetz bestimmten Grenzen nach Belieben seine Sache gebrauchen, nutzen, über sie verfügen und die Einwirkungen anderer Personen ausschließen".16

Nach der herrschenden Zivilrechtsrechtslehre wird auch die Vertragsfreiheit verfassungsrechtlich garantiert.17 Und schließlich werden im Gesellschaftsrecht auch unterschiedliche, den Erfordernissen der Organisationsfreiheit des Unternehmers Rechnung tragende Rechtsformen der Unternehmensorganisation angeboten, die dem deutschen Recht vergleichbar sind. 18

Andererseits darf das Eigentum nach Art. 23 Verf durch Gesetz beschränkt werden, um eine Verletzung der Freiheit anderer zu verhindern, eine drohende Krise abzuwenden, die gesellschaftliche Ordnung aufrechtzuerhalten oder zur Mehrung des öffentlichen Nutzens.

Die Unternehmerfreiheit wird darüber hinaus noch durch verfassungsrechtliche Politikziele beeinflußt. Die Verfassung der Republik China enthält nämlich eine stark einseitige Ausrichtung auf die politische Ideologie der KMT.19 Bereits in der Präambel wird betont, daß die Verfassung entsprechend der "Hinterlassenen Lehre" von Sun Yat-sen ausgearbeitet worden sei. Nach Art. 1 Verf beruht die Republik China auf den Drei Volksprinzipien, beziehungsweise den Drei Lehren vom Volke (Sanminzhuyi), nämlich dem Nationalismus, den Rechten des Volkes und der Volkswohlfahrt.20 Der Hauptinhalt der Lehre der Volks-

14 Lin Jidong, Band I, Art. 15, S. 252.

15 So Lin Jidong, ebenda, Band I, Art. 15, S. 249 und für Deutschland BVerfGE 50, 290 ff. (339).

16 Zitiert nach Karl Bünger, Zivil- und Handelsgesetzbuch sowie Wechsel- und Scheckgesetz von China, Marburg, 1934, S. 216.

17 Wang Zejian, Zhaibian zong lun (Schuldrecht Allgemeiner Tei), Taipei 1988, S. 13.

18 Siehe zusätzlich zu dem bereits erwähnten Zivilgesetzbuch noch das am 26.12 .1929 verkündete Gesellschaftsgesetz (Gongsi fa).

$19 \mathrm{Zu}$ dem über achtzigjährigen Kampf chinesischer Demokraten für einen verfaßten Rechtsstaat siehe Wolfgang Lasars, Die Machtfunktion der Verfassung - Eine Untersuchung zur Rezeption von demokratisch-rechtsstaatlichem Verfassungsrecht in China, in: JöR (1993).

20 Zu den Drei Volksprinzipien im verfassungsrechtlichen Sinne siehe Lin Jidong, Zhonghua minguo xianfa shilun (Interpretation der Verfassung der Republik China), Taipei, 31. Aufl. 1977, S. 122 f. 
wohlfahrt sei es, eine gleichmäßige Befriedigung der Staatsfinanzen und des Volkswohls zu erreichen. Weiterhin sei mittels des Grundsatzes "der gleichmäßigen Rechte an Grund und Boden" der höchste Nutzungswert des Bodens zu erreichen und eine Bodenkonzentration zu verhindern und mit dem Grundsatz der "Beschränkung des Kapitals" eine übermäßige Konzentration an privatem Kapital zu verhindern.

Im Abschnitt über die Volkswirtschaft wird dann das Verfassungsprinzip der Volkswohlfahrt als das Hauptprinzip der Wirtschaftspolitik näher beschrieben:

Art. 142: "In der Volkswirtschaft ist die Lehre der Volkswohlfahrt als Grundprinzip zugrundezulegen und die (Politik) der gleichmäßigen Rechte an Grund und Boden und der Beschränkung des Kapitals durchzuführen, um damit eine gleichmäßige Befriedigung der Staatsfinanzen und des Volkswohls anzustreben".

Die Politik der gleichmäßigen Rechte an Grund und Boden und der Beschränkung des Kapitals seien hierbei die Mittel der Lehre der Volkswohlfahrt, deren Ziel in der gleichmäßigen Befriedigung der Staatsfinanzen und des Volkswohls liege.21

Art. 144: "Offentliche Einrichtungen und andere monopolartige Unternehmen sind prinzipiell öffentlich zu betreiben, nach Genehmigung aufgrund von Gesetzen können sie auch von Bürgern betrieben werden".

In der national-chinesischen Verfassungslehre werden "Öfentliche Einrichtungen" auf "elektrisch Licht, Telefon, Kraftwagen, Eisenbahn und so weiter" bezogen, die die Offentlichkeit benutzen müsse. "Andere monopolartige Unternehmen" beziehe sich auf auf Unternehmen mit großem Kapitalbedarf, wie zum Beispiel "Stahlschmelzen".22 Die Hauptbedeutung des Zieles der "Beschränkung des Kapitals" liege in der Entwicklung von Staatskapital und der Beschränkung von Privatkapital, um auf diese Weise die Schwächen des Kapitalismus zu korrigieren.23

Art. 145: "Privater Reichtum und private Unternehmen, von denen der Staat denkt, daß sie die ausgeglichene Entwicklung der Staatsfinanzen und des Volkswohls behindern, sind aufgrund von Gesetzen zu beschränken.

Genossenschaften sind vom Staat zu fördern und zu unterstützen.

21 Lin Jidong, Zhonghua minguo xianfa zhu tiao shiyi (Kommentar zur Verfassung der Republik China), Taipei, Band IV, 5. Aufl. 1990, Art. 142, S. 274.

22 Lin Jidong, ebenda, Band IV, Art. 144, S. 284, Guan Ou, Zhonghua minguo xianfa lun(Die Verfassung der Republik China), Taipei 4. Aufl. 1981, S. 298.

23 So Lin Jidong, Zhonghua minguo xianfa zhu tiao shiyi (Kommentar zur Verfassung der Republik China), Band IV, Art. 144, S. 284. 
Die Produktionsbetriebe der Bürger und der Außenhandel sind vom Sta ıt zu fördern, anzuleiten und zu schützen".

In der Kommentierung wird dazu ausgeführt, daß die Vermehrung privaten Reichtums und die Entwickung privater Unternehmen grundsätzlich auch zum gesellschaftlichen Reichtum beitragen und die Entwicklung der Volkswirtschaft fördern könne, sie würden deswegen auch vom Staat gefördert und geschützt werden. Wenn aber der private Reichtum und die Macht privater Unternehmen übermäßig würden und die Entwicklung zur gleichmäßigen Befriedigung der Staatsfinanzen und des Volkswohls behindern, seien sie durch Gesetze zu beschränken, allerdings nicht über das notwendige Maß hinaus, die Schranke sei eng auszulegen. 24

Der Hauptinhalt der Aufnahme von die Wirtschaftspolitik betreffenden Artikeln liege nach der Verfassungslehre in der Beschränkung der Eigentumfreiheit und Handelsfreiheit.25 Allerdings wiesen die von der Verfassung normierten "Grundlegende Politiken" nur auf Ziele hin, nach denen sich die Regierung ausrichten solle. Sofern ein Ziel noch nicht erreicht worden sei, könne man nicht von einem Verfassungsverstoß reden. 26 Im übrigen werden von der konservativen, national-chinesischen Verfassungslehre diesbezüglich lediglich formelhaft, geradezu gebetsmühlenartig, einheitlich, bestimmte politische Formulierungen von Sun Yat-sen wiederholt, ohne daß ein Bemühen um weitere verfassungstheoretische Ausarbeitung, Vertiefung oder Prüfung ihrer rechtlichen oder tatsächlichen Umsetzung erkennbar ist. 27

Zusammengefaßt läßt sich festhalten, daß in der Verfassung der Republik China grundsätzlich die Unternehmerfreiheit garantiert wird. Im Folgenden wird nun der Frage nachzugehen sein, wie vier Jahrzehnte der Alleinherrschaft der KMT auf die Wirtschaftsverfassung von Taiwan gewirkt haben beziehungsweise noch wirken, um so die Reformforderungen nach einer Liberalisierung zu verstehen.

24 Lin Jidong, ebenda, Band IV, Art. 145, S. 285 ff.

25 Lin Jidong, ebenda, Band IV, Abschn. 3 Volkswirtschaft, S. 269.

26 Guan Ou, Zhonghua minguo xianfa lun(Die Verfassung der Republik China), S. 291.

27 Vergleiche etwa nur die Ausführungen zum Verfassungsabschnitt Volkswirtschaft in Guan $O u$, Zhonghua minguo xianfa lun(Die Verfassung der Republik China), S. 295 ff; Liu Qingrui, Zhonghua minguo xianfa yaoyi(Grundzüge der Verfassung der Republik China), Taipei 9. Aufl. 1976, S. 249 f.; Hong Yingzao, Zhonghua minguo xianfa xin lun (Lehrbuch zur Verfassung der Republik China), Taipei 7. Aufl. 1974, S. 263 f. 


\section{Einschränkungen der Unternehmerfreiheit}

Die Unternehmerfreiheit kann auf verschiedenste Weise eingeschränkt werden. Grob eingeteilt kann sie durch eingreifende und beschränkende Gesetze, durch staatliche Konkurrenzwirtschaft und noch stärker durch staatliche oder staatlich angeordnete Monopole eingegrenzt werden. 28

Bei der folgenden Untersuchung der Einschränkungen der Unternehmerfreiheit ist gezielt auf die Besonderheiten der Wirtschaftsverfassung Taiwans, die Unterschiede zu liberalen Wirtschaftsverfassungen in demokratischen Staaten einzugehen. Einleitend ist diesbezüglich zu erinnern, daß die von der KMT geführte Nationalregierung nach ihrer Niederlage auf dem chinesischen Festland nach Taiwan geflohen ist und dort von 1949 bis 1987 mit Kriegsrecht herrschte.29

\subsection{Einschränkungen durch Sondergesetze}

Seit Jahrzehnten hat sich die Regierung der KMT größtenteils bis 1992 fortgeltende, extrem weitreichende Vollmachten und Eingriffsmöglichkeiten vorbehalten. Beispielhaft sei hier nur auf folgende zwei Wirtschaftsgesetze hingewiesen.

Im Jahre 1942 hatte die Nationalregierung das "Gesetz der nationalen Generalmobilmachung" erlassen. 30 Nach $\S 1$ ist dieses Gesetz zum Zwecke des konzentrierten Einsatzes der personellen und materiellen Kräfte des ganzen Landes zur Durchführung des Widerstandskrieges gegen Japan erlassen worden. 1945 kapitulierte Japan, dennoch hat die KMTRegierung an diesem Gesetz bis 1992 festgehalten.

Der Bereich dieses Gesetzes umfaßt neben militärischen Gütern auch Lebensmittel, Kleidung, medizinische Güter, Schiffe, Kraftwagen und andere Transportmittel, Baumaterial, Strom und Gas und alle vorübergehend von der Regierung zusätzlich einbezogene Güter.

Ossenbühl, AöR 115 (1990), S. 13 f.

29 Die Taiwanesische Gesellschaft zur Förderung der Menschenrechte kritisierte diese Politik als die mit über 38 Jahren längsten Kriegsrechtsverkündung der Welt, in: Taiwan 1987 - 1990 Renquan baogao (Taiwan 1987 - 1990 Bericht über Menschenrechte), herausgegeben von Taiwan renquan cujin hui (Taiwanesische Gesellschaft zur Förderung der Menschenrechte), Taipei, 1990, S. 17; das Kriegsrechtsgesetz (Jieyan fa) ist nach Aufhebung des Kriegsrechts in 1987 durch das "Gesetz für Nationale Sicherheit während der Periode der Periode der Mobilmachung zur Niederschlagung der Rebellion" (Dongyuan kanluan shiqi guojia anquan fa) ersetzt worden.

30 Guojia zongdongyuan fa vom 29. März 1942. Einen detaillierten Überblick über dieses Gesetz und die zahlreichen auf ihm beruhenden Verordnungen gibt Hu Kaicheng, Guojia zongdongyuan fa gailun (Das Gesetz der nationalen Generalmobilmachung), Taipei, 1962. 
Produktion, Verkauf, Verwendung, Reparatur, Lagerung, Konsum, Transport und Weitergabe dieser Güter können von der Regierung im Bedarfsfalle angewiesen, beaufsichtigt, beschränkt und verboten werden. Ebenso können deren Handelspreise und -mengen kontrolliert werden. Nach Alter, Eignung u.s.w. können Arbeitskräfte verteilt werden, die Anzahl der Beschäftigten in Betrieben beschränkt und Streiks verboten werden. Neugründungen oder Zusammenschlüsse von Unternehmen, Kapitalerhöhungen, Kreditaufnahmen u.s.w. können beschränkt werden. Die Verletzung dieses Gesetzes und der auf ihm beruhenden Verordnungen ist unter Strafe gestellt.

Im Jahre 1951 verkündete die Regierung sogar noch eine "Verordnung zum Erlaß von Verordnungen und Verwaltungsanweisungen gemäß dem Gesetz der nationalen Generalmobilmachung während der Periode der Niederschlagung der Rebellion".31 Darin wird das Gesetz der nationalen Generalmobilmachung als das "grundlegende, große Gesetz" (jinben da fa) der Regierung bei der Wahmehmung ihrer Aufgaben bezeichnet und hinzugefügt, $\mathrm{da} ß$ die Regierung dabei nicht an Verfassungsnormen gebunden sei.32 Uber zwanzig Verordnungen wurden auf dieser Grundlage erlassen. Hervorzuheben ist hierbei die strenge Kontrolle der Ein- und Ausfuhr von Devisen. 33

Ein weiteres Wirtschaftsgesetz, welches das Wirtschaftsministerium mit äußerst weitreichenden Vollmachten ausstattet, ist das "Gesetz zur Kontrolle von Landwirtschaft, Bergbau, Industrie und Handel während der außerordentlichen Zeit" aus dem Jahre 1938.34 Das Wirtschaftsministerium kann danach betreffend die Methode der Produktion und des Betriebes, der Arten und der Menge von zu bevorratenden Rohstoffen, der Arbeitszeiten und des Arbeitsentgeltes, der Produktqualität, der Produktionsmenge und der Lagermenge, der Produktionskosten u.s.w. "angemessene Standards" (shidang zhi biaozhun) festsetzen. Entsprechend den örtlichen Bedürfnissen kann die Regierung eigene Unternehmen betreffend Gegenstände des täglichen Bedarfs betreiben. Vom Wirtschaftsministerium bestimmte Betriebe dürfen ohne Genehmigung dieses Ministeriums nicht den Betrieb unterbrechen oder einstellen, sollte deren Finanzkraft für eine Wiederaufnahme nicht ausreichen, können diese Betriebe verstaatlicht werden. Weiterhin dürfen bei vom Ministerium bestimmten Betrieben oder Produkten die Unternehmer kein "spekulatives, monopolartiges oder sonst (den Markt) manipulierendes Verhalten" zeigen. Betreffend den Im- oder Export bestimm-

31 Kanluan shiqi yi guojia zongdongyuan fa banfa fagui mingling banfa vom 8.12.1951.

32 Allerdings machen mittlerweile auch jüngere Parlamentarier der KMT Zweifel an der Verfassungsmäßigkeit dieses Rechtssystems der Generalmobilmachung geltend und fordem die sofortige Abschaffung, siehe Lianhe bao vom 22.5.1991.

$33 \mathrm{Ab}$ dem 15.7.1987 sind nach einer Verfügung des Verwaltungshofes, also der Regierung, die insbesondere Import- und Export betreffenden Devisenkontrollen nicht mehr anzuwenden. Zum selben Zeitpunkt hob die Zentralbank zwölf Devisenverordnungen auf.

34 Feichang shiqi nong kuang gong shang guanli tiaoli vom 6. Oktober 1938, dessen $\S 1$ noch am 1. Juni 1973 (sic!) geändert worden ist. 
ter Waren darf das Wirtschaftsministerium Beschränkungen oder Verbote verhängen u.s.w. Häufig sind Verstöße gegen dieses Gesetz strafbar.

Bereits an diesen beiden Beispielen wird deutlich, daß sich die Regierung der KMT in Taiwan über vier Jahrzehnte lang das ganze Instrumentarium einer kriegsrechtlichen Zwangsbewirtschaftung vorbehalten hat und somit die Unternehmerfreiheit und zwar im gesamten, verfassungsrechtlich geschützten Bereich erheblichen Einschränkungen und vor allen Dingen großen Unsicherheiten und Unwägbarkeiten ausgesetzt hat.

Sucht man nach Gründen für dieses Festhalten an den Sondernormen so fällt auf, daß die Regierung von Chiang Kai-shek in der Gemeinsamen Erklärung der Regierungen der Republik China und der Vereinigten Staaten vom 23. Oktober 1958 zugesichert hatte, die Wiedervereinigung Chinas auf die "Durchführung der Drei Volksprinzipien von Dr. Sun Yat-sen und nicht auf Waffengewalt" zu stützen. Nach außen war die KMT-Regierung von einer militärischen Rückeroberung, die sowieso unmöglich gewesen wäre, bereits frühzeitig abgerückt. 35 Nach innen hingegen hielt sie am Sonderrecht fest. Die Gründe für das Aufrechterhalten der wirtschaftlichen Sondergesetze liegen weniger in einer permanenten, direkten Wirtschaftskontrolle. Hauptsächlich sind sie als Teil des allgemeinen Kriegsrechts zu verstehen, daß dazu diente, die politischen Teilhaberechte der taiwanesischen Mehrheit außer kraft zu setzen und so die herrschende, vom chinesischen Festland stammende Flüchtlingselite der KMT ohne Wahlen an der Macht zu halten.

\subsection{Einschränkungen durch Staatsunternehmen}

Der Aufbau von staatlichen Unternehmen stellt einen viel stärkeren, weil unmittelbaren Eingriff in die verfassungsrechtlich garantierte Unternehmerfreiheit dar.

Die historische Entwicklung der staatlichen Wirtschaft in Taiwan kann wie folgt skizziert werden: Nach dem Zweiten Weltkrieg hat die Nationalregierung der KMT gemäß der "Verordnung über die Verfahrensweise betreffend Unternehmen von Feinden im wiedererlangten Gebiet"36 sämtliche Unternehmen, die Japan in Taiwan besaß, übernommen. Diese Unternehmen umfaßten die wichtigen Wirtschaftsbereiche Finanzen, Verkehr und Transport, industrielle Rohstoffe, Metallverarbeitung, Bergbau, Maschinenbau, Düngemittel, Zement, Papier, Wasserkraft und Elektrizität und in der Landwirtschaft Zucker, Salz, Tabak, Alkohol und Waldwirtschaft. Vor dem Krieg gab es in Taiwan 530.000 Unternehmen mit einem Kapital von über 200.000 (damaligen) Yen, davon hatten taiwanesische

35 Yin Haiguang, Ziyou zhongguo zhi lu (Der Weg des freien China) in: Quanji (Werke) Band 12 Zhengzhi yu shehui (Politik und Gesellschaft), S. 771 ff. (773).

36 Shoufu qu diwei shangye chuli banfa. 
Unternehmen einen Anteil nur von 8,3\% mit einem Kapitalanteil von $9 \%$; über $90 \%$ der japanischen Unternehmen waren überwiegend in privater Hand. 37

Diese Privatunternehmen verstaatlichte die Nationalregierung einseitig gegen den - worauf unten noch einzugehen sein wird -erbitterten Protest der Taiwanesen. 1949 wurden einige wenige staatliche Betriebe vom Festland nach Taiwan transferiert. Insgesamt aber läßt sich sagen, daß außer landwirtschaftlichen Kleinbetrieben und lokalen Unternehmen in Industrie und Handel die von den Japanern übernommenen Unternehmen die gesamte Wirtschaft Taiwans beherrschten. Nach der Verstaatlichung haben sie das Rückgrat des staatlichen Unternehmensbereiches in Taiwan gebildet.

Anfang der fünfziger Jahre wurden einige staatliche Unternehmen zur Finanzierung der Bodenreform privatisiert, im Prinzip aber haben die vom chinesischen Festland geflohenen KMT-Politiker am System der Staatsunternehmen festgehalten. In den sechziger Jahren entwickelte sich eine private Exportindustrie insbesondere in den Bereichen Textil, Plastik und Elektronik. Die taiwanesische Privatwirtschaft wuchs in dieser Zeit sehr stark, andererseits baute die Regierung Anfang der siebziger Jahre in den Bereichen der petro-chemischen Industrie und zwar insbesondere im Bereich der von der Exportindustrie als Rohstoffe benötigten Zwischengüter und im Schiffsbau neue öffentliche Unternehmen auf.38

Betreffend den Bereich neugegründeter, staatlicher Unternehmen ist es außerhalb des Finanzbereiches vor allem das Militär, welches in den Bereich der Privatwirtschaft eingedrungen ist. Es bedient sich dabei der öffentlich-rechtlichen "Unterstützungsvereinigung ausgeschiedener Offiziere und Soldaten", welche zahlreiche monopolartige oder sonst privilegierte Unternehmen betreibt. 39 Die Wirtschaftstätigkeit dieser Unterstützungsvereinigung umfaßt die Landwirtschaft, die industrielle Weiterverarbeitung landwirtschaftlicher Erzeugnisse, den Bergbau, die Metallverarbeitung, die infrastrukturelle Erschließung neuer Gebiete, Baustoffe, Land- und Seetransport, die chemische Herstellung von Arzeneimitteln, die Papierherstellung, Druckindustrie, Verlage, Elektronik, militärische Güter u.s.w. und monopolisiert das Gasangebot von ganz Taiwan. 40

37 Angaben nach Chen Shimeng u.a., Jiegou dang guo zibenzhuyi/Disintegrating KMT-State Capitalism, Taipei, 1991, S. 28; siehe dazu auch Zhu Yunhan, Guazhan jingji yu weiquan zhengzhi tizhi (Das System aus oligopolisierter Wirtschaft und autoritärer Politik) in: Longduan yu boxue Weiquanzhuyi de zhengzhi jingji fenxi (Monopolisierung und Ausbeutung - Eine politisch-wirtschaftliche Analyse eines autoritären Regimes), hrsg. v. Taiwan yan jiu jijinhui (Forschungsstiftung Taiwan), Taipei, 1989, S. 145.

38 Chen Shimeng u.a., Dang guo zibenzhuyi/KMT-State Capitalism, S. 34.

39 Diese Vereinigung ist aufgrund des "Gesetzes über die Organisation der Unterstützungsvereinigung ausgeschiedener Offiziere und Soldaten des Verwaltungshofes" (Xingzhengyuan guojun tuichuyi guan bing fudao wei yuanhui zuzhi tiaoli) vom 27.12.1955 gegründet worden.

40 Zhang Qingxi, Gongying yu dangying shiye de duzhan: Taiwan zhengjing de yan(Die Monopolisierung durch öffentliche Untemehmen und Untemehmen der Partei: Das polit-ökonomische 
Nach $\S 8$ des "Gesetzes über die Unterstützung ausgeschiedener Offiziere und Soldaten" 41 kann die Regierung bei allen Bauprojekten, wie zum Beispiel bei Wasser-, Straßen-, Eisenbahn-, Brücken-, Hafen- und Gebäudebauprojekten und beim Bau militärischer Anlagen, das von dieser Soldaten-Unterstützungsvereinigung gegründete Bauunternehmen vorrangig beauftragen und zwar zu "vereinbarten Preisen". Letzteres stellt ein weiteres Privileg gegenüber Privatunternehmern dar, da diese sich über öffentliche Ausschreibungen Konkurrenzpreisen stellen müssen.

Darüber hinaus gibt es noch den Bereich verdeckt staatlicher beziehungsweise öffentlicher Unternehmen. Nach der engen Legaldefinition von $\S 3$ Gesetz über die Kontrolle von staatlichen Unternehmen 42 und $\S 2$ Gesetz über die Privatisierung von öffentlichen Unternehmen 43 sind Unternehmen nur "staatliche" beziehungsweise "öffentliche" Unternehmen, wenn die Regierungsbeteiligung mindestens über $50 \%$ beträgt, wobei die Beteiligung nach einzelnen Behörden gerechnet wird.44 Eine Vielzahl von scheinbar privaten Unternehmen wird aber tatsächlich vom Staat und - worauf unten noch einzugehen sein wird - der KMT kontrolliert. Thre Anteile sind auf verschiedene staatliche Unternehmen, Regierungsbehörden, der Gesellschaft zur Rettung des Vaterlandes, mit öffentlichen Geldern gegründeter Stiftungen und Parteiunternehmen verteilt, so daß sie nicht als öffentlich erscheinen. 45

Wie verwoben und dunkel die Eigentumsverhältnisse in diesem Bereich sind mag an folgendem Beispiel gezeigt werden: Als die Republik China 1971 die Vereinten Nationen verließ, befürchtete die KMT-Regierung, daß der im Ausland angelegte Teil des Staatsvermögens von der Regierung in Peking übernommen werden könnte. Deswegen beauftragte sie die privatrechtlich organisierte aber der Partei KMT gehörende Zentrale Investitionsgesellschaft (Zhongyang touzi gongsi) mit der Verwahrung und Verwaltung. Durch Gesetz vom 15.12.1971 wurde nun die staatliche China Bank (Zhongguo yinhang) privatisiert zur International Commercial Bank of China (Zhongguo guoji shangye yinhang), was die Regierung und die der KMT nahestehende Presse als großen, für die Wirtschaft bedeutungsvollen Privatisierungserfolg herausstellte. Tatsächlich aber wurden lediglich von der Zentralen Investitionsfirma einige ihr nahestehende Parteimitglieder der KMT unter Verstoß gegen mehrere Wirtschaftsgesetze als Strohmänner "arrangiert". Entgegen dem aus-

Krebsgeschwür Taiwans, in: Quan guo minjian jingji huiyi shilu(Nationale private Wirtschaftskonferenz), hrsg. v. Demokratische Fortschrittspartei, Band 2, Taipei, 1991, S. 28 f.

41 Guojun tuichuyi guan bing fudao tiaoli vom 15.5.1964.

42 Guoying shiye guanlifa vom 20.1.1949.

43 Gongying shiye yizhuan minying tiaoli vom 26.1.1953.

44 Nach modemer Ansicht in der taiwanesischen Wirtschaftswissenschaft sind hingegen alle Unternehmen staatlich, in denen der Staat einen beherrschenden Einfluß ausüben kann, Chen Shimeng u.a., Dang guo zibenzhuyi/KMT-State Capitalism, S. 21.

45 Chen Shimeng u.a., Dang guo zibenzhuyi/KMT-State Capitalism, S. 65 mit Beispielen. 
drücklichen Wortlaut des Privatisierungs gesetzes werden tatsächlich über $60 \%$ der Aktien von der staatlichen Entwicklungsstiftung (Xingzhengyuan kaifa jijinhui) gehalten. 46

Ein von taiwanesischen Wirtschaftswissenschaftlern durchgeführter Vergleich mit zehn westlichen und damals sozialistischen Staaten kommt zu folgendem Ergebnis:

"Die Wirtschaftsstruktur Taiwans weist unter den Staaten mit einer freien Wirtschaftsstruktur eine Besonderheit auf, nämlich die, daß sie nicht der eines Staates mit freier Wirtschaftsstruktur entspricht". Wenn man über die enge Legaldefinition hinausgehe und noch die anderen staatlich beherrschten Unternehmen einbeziehe, "dann - so ist zu befürchten - daß das in der Welt für seinen Antikommunismus berühmte Taiwan unter den "antikommunistischen Staaten" den kommunistischen Staaten am nächsten kommt".47

\subsection{Einschränkungen durch Parteiunternehmen}

Neben den oben beiden genannten Beschränkungen ist die Unternehmerfreiheit - im Unterschied zu liberalen Wirtschaftsverfassungen in demokratischen Staaten - zusätzlich noch durch folgendes Phänomen beeinträchtigt: die Parteiunternehmen der über vierzig Jahre lang ohne demokratische Wahlen herrschenden KMT.48

Erst gegen Ende der achtziger Jahre nach Aufhebung des Kriegsrechts sind zunächst von der wirtschaftlichen Fachpresse Taiwans erste Untersuchungen zu dem dunklen und bis heute noch nicht voll erhellten Wirtschaftsbereich "Parteiunternehmen der KMT" durchgeführt worden.49 Nach bisherigen Erkenntnissen unterstehen der Zentralen Finanzkommission der KMT (Zhongyang caiwu weiyuanhui) zwölf reine Parteiunternehmen im Finanz-, Versicherungs-, Elektronik- und Baubereich. Dazu gehören auch die oben bereits erwähnte Zentrale Investitionsgesellschaft und das Finanzinstitut Guanghua touzi, die ihrerseits an

Siehe den Hintergrundbericht von Liang Yonghuang mit dem bezeichnenden Titel "Staatsvermögen, Parteivermögen und Privatvermögen ungetrennt?" (Guochan, dangchan, sichan bu fen ?) in: Caixun Nr. 86 (Mai 1989), S. 105 ff.

47 Chen Shimeng u.a., Dang guo zibenzhuyi/KMT-State Capitalism, S. 95.

$48 \mathrm{Zu}$ den jetzt einsetzenden Reformen hin zu einem zukünftigen, demokratischen Rechtsstaat siehe Wolfgang Lasars, "Rückkehr zur Verfassung, Reform der Verfassung oder Erlaß einer neuen Verfassung? - Ein Zwischenbericht über die demokratische Reform im national-chinesischen Verfassungsrecht", VRÜ 25 (1992), S. 115-160.

49 Die frühesten Untersuchungen in: Caixun Nr. 74 (Mai 1988), Dangying shiye zhuanhao (Sondernummer Parteiuntemehmen), S. 113-202; Zhuoyue zazhi März 1989, S. 36 ff. und Shangye zhoukan/Business Weekly Nr. 109 (25.12.1989), S. 22-62. 
sechzig bis siebzig Unternehmen häufig mit Monopolstellung, wie etwa die Börse Taiwans, oder marktbeherrschender Stellung beteiligt sind. 50

Nachdem sich dann die taiwanesische Wissenschaft dieses Themas angenommen hatte, mußte auch die KMT Stellung nehmen. Anfang Januar 1992 bezifferte der Vorsitzende der Zentralen Finanzkommission der KMT Xu Lide den Gesamtbetrag gewerblicher Investitionen der KMT mit 15 Milliarden NT\$, was einem Betrag von etwa einer Milliarde DM entspricht, ohne allerdings diesen Betrag aufzuschlüsseln.51 Andererseits wird dem in der Offentlichkeit entgegengehalten, daß die beiden oben genannten Finanzinstitute über Anteile an börsengängigen Unternehmen gemessen an den Börsenpreisen in Höhe von 47,8 Milliarden NT\$ verfügten, was etwa 3,2 Milliarden DM entspricht, und wenn man dann noch die nicht börsengängigen Anteile hinzurechne, komme man auf einen Betrag von über 100 Milliarden NT\$, was einem Wert von über 6,7 Milliarden DM entspricht.52 Eine andere Schätzung von Wirtschaftswissenschaftlern kommt noch zu einem wesentlich höheren Ergebnis. 53

Viele Einzelheiten über die unternehmerischen Aktivitäten der KMT sind noch unbekannt, dennoch haben bisherige Untersuchungen drei Besonderheiten festgestellt: 54

Erstens bevorzugt die KMT Investitionen in oder mit staatlichen Unternehmen. Ein Grund für die Verknüpfung von Parteiunternehmen mit staatlichen Unternehmen wird darin gesehen, daß politische Privilegien wirtschaftliche Sicherheit garantieren. Dies gelte insbesondere für Investitionen in von der Regierung besonders finanziell oder wissenschaftlichtechnisch geförderten Branchen wie der petro-chemischen Industrie.55 Im übrigen müsse die Regierung bei Staatsunternehmen Falle eines wirtschaftlichen Rückschlages einspringen, so daß ein Konkursrisiko vermieden werde.

50 Siehe Li Naixiong, Dangying shiye jin xi (Die Parteiunternehmen, heute und früher), in: Zhuoyue zazhi März 1989, S. 37 und die Aufstellung in: Chen Shimeng u.a., Dang guo zibenzhuyi/KMTState Capitalism, S. $80 \mathrm{f}$.

51 Im Interview mit der Wirtschaftszeitung Jingji ribao vom 6.1.1992.

52 Siehe die Aufstellung in der konservativen Tageszeitung Zhongguo shibao vom 23.1.1992.

53 Chen Shimeng u.a., Dang guo zibenzhuyi/KMT-State Capitalism, S. 70.

54 Liang Yonghuang, Guomindang shi chaoji dafu weng! (Die KMT ist ein Superreicher!), in: Caixun Nr. 92 (November 1989), S. 55 ff. mit einer detaillierten Grafik der wirtschaftlichen Verflechtungen der KMT; Chen Shimeng u.a., Dang guo zibenzhuyi/KMT-State Capitalism, S. 69 ff.

55 Kritisch ist darauf hingewiesen worden, daß die Wirtschafts- und Finanzspezialisten der KMTRegierung gehalten seien, während sie die Wirtschaftspolitik konzipieren, auch immer für die Parteikasse neue Investitionsmöglichkeiten zu schaffen. Aus diesem Grunde seien Parteiuntemehmen immer mit den nach 1970 neu vorangetriebenen strategischen Industrien oder Staatsbetrieben, denen sich die staatlichen Forschungseinrichtungen widmeten, verbunden worden, siehe Zhu Yunhan, Guazhan jingji yu weiquan zhengzhi tizhi (Das System aus oligopolisierter Wirtschaft und autoritärer Politik) in: Longduan yu boxue_(Monopolisierung und Ausbeutung), S. 150. 
Zweitens bevorzugt die KMT Investitionen in Unternehmen mit monopolartiger Stellung, insbesondere privilegierte Unternehmen im Bereich des Finanz- und Versicherungsgewerbes sowie im Wertpapierhandel. So gehörte der KMT das 1959 gegründete Finanzinstitut Zhonghua kaifa xintuo touzi gongsi, das damals einzige Institut für mittel- und langfristige Kredite in Taiwan, welches bei nur geringem eigenen Kapital unter anderem die Weitervergabe von Krediten monopolisierte, die auf der US-Wirtschaftshilfe beruhten. Weiterhin ist die KMT an der Börse Taiwans beteiligt. Von den früh vor der Aktienhausse besonders genehmigten vier Wertpapierhäusern gehören zwei, Guanghua und Zhonghua, der KMT.

Und drittens schließlich bevorzugt die KMT Investitionen im Bereich der Medien, so etwa gehören der KMT die Nachrichtenagentur Taiwans Zhongyang tongxun she, die Rundfunkanstalt Zhongguo guangbo gongsi und eine der drei Femsehanstalten Taiwans, die Zhongguo dianshi gongsi, sowie mehrere Parteizeitungen.56

Hinzu kommt noch seit 1988 eine Politik der KMT, direkt in profitable private Großunternehmen zu investieren. Fast alle privaten Großunternehmen sind so auch zu Wirtschaftspartner der KMT geworden, die auf diese Weise am Erfolg taiwanesischer Privatunternehmer partizipiert.

Schließlich gibt es noch die formal private "Anti-kommunistische Organisation der Jugend zur Rettung des Vaterlandes".57 Diese Organisation, der jahrzehntelang fast die gesamte Jugend Taiwans angehörte, wurde bereits in den fünfziger Jahren aus liberaler Sicht kritisiert, weil sie lediglich ein Propagandainstrument der KMT zur Indoktrinierung der Jugend sei.58 Die Organisation zur Rettung des Vaterlandes betreibt in ganz Taiwan hotelartige Betriebe auf in der Regel öffentlichem Land privilegiert ohne Mietaufwendungen sowie einen Verlag.

Zwischen Staat und KMT besteht eine doppelte Verbindung. Einmal besteht eine Verbindung zwischen Parteikasse und Fiskus: Mittels öffentlicher Aufträge an Parteiunternehmen werden öffentliche Gelder in die Parteikasse geleitet, insbesondere aber nicht ausschließlich im Medienbereich etwa durch Kauf von Sendezeit beim Parteisender oder kostenlose Ver-

56 Diese Unternehmen wurden von dem langjährigen Vorsitzenden der Zentralen Finanzkommission $Y u$ Guohua Anfang der siebziger Jahre ausgegliedert und unterstehen seitdem der Zentralen Kulturabteilung (Zhongyang wenhua gongzuohui).

57 Zhongguo qingnian fangong jiu guo tuan, siehe dazu Chen Shimeng u.a., Dang guo zibenzhuyil KMT-State Capitalism, S. 72.

58 Qingnian fangong jiu guo tuan wenti (Das Problem der Anti-kommunistischen Organisation der Jugend zur Rettung des Vaterlandes) in Ziyou zhongguo (Freies China) Band 18 Heft 1 (1.1.1958), S. 5 ff. Es wurde deren Auflösung gefordert. 
teilung von Parteizeitungen an im Ausland Studierende ${ }^{59}$ oder es werden etwa Gebäude der Partei von Regierungsstellen angemietet. Seit Jahren ist dies bei den Haushaltsberatungen ein Kritikpunkt der Parlamentarier, teilweise sogar auch der der KMT.

Weiterhin besteht häufig eine Personalunion auf den wirtschafts-und finanzpolitischen Leitungsebenen von Staat und KMT. So war beispielsweise der ehemalige Präsident der Zentralbank und spätere Vorsitzende des Council for Economic Planning and Development Yu Guohua auch gleichzeitig Vorsitzender der Zentralen Finanzkommission der KMT. Es war Yu Guohua, der seit Anfang der siebziger Jahre während seiner über zehnjährigen Vorstandstätigkeit in der Finanzkommission das moderne Wirtschaftsimperium der KMT aufbaute. Danach ist der ehemalige Wirtschaftsminister $X u$ Lide gefolgt, der nun der Zentralen Finanzkommssion vorsteht.60 Die herausragende Stellung innerhalb der Regierung gab diesen Finanzspezialisten der KMT die erforderlichen Informationen zum Aufbau des Wirtschaftsimperiums der Partei. Die Finanzkommission beaufsichtig die Parteiunternehmen, plant und genehmigt die Ausgaben der Partei und so weiter und ist aus diesem Grunde von der Wirtschaftspresse die eigentliche "Lebensader der KMT" genannt worden.61 Zusätzlich hat die KMT im Januar 1992 noch eine neue Finanzabteilung (Jinrong dangbu) eingerichtet, in der alle Parteimitglieder in öffentlichen und privaten Finanzunternehmungen organisiert sind. Diesem speziellen Leitungssystem der Partei steht der jetzige Finanzminister Wang Jianxuan vor, was im Frühjahr 1992 zu heftiger Kritik im Parlament führte.62

Die Herkunft des Reichtums der KMT ist noch dunkler als ihr Umfang. Hier wird die Wichtigkeit einer öffentlichen Rechenschaftspflicht von Parteien deutlich, wie sie gemäß Art. 21 Abs. 1 Satz 3 GG normiert ist. Bezeichnenderweise hat der Innenminister der KMT diesbezüglich es abgelehnt, ein bisher in Taiwan nicht vorhandenes Parteiengesetz auszuarbeiten. 63

Da sich einerseits die KMT weigert, Auskunft über die Herkunft ihres Reichtums zu geben, und sonst keine überprüfbaren Unterlagen vorhanden sind, sollen im Folgenden nur die kritischen Hinweise in der liberalen, taiwanesischen Öfentlichkeit wiedergegeben werden:64 In der Anfangszeit hätte danach die KMT die Devisenkontrolle als ein Instrument benutzt, um sich illegal zu bereichern. Man hätte von taiwanesischen Unternehmern Gelder

59 Zhang Junhong, Taiwan gongshangren (Taiwanesische Unternehmer), Taipei, 1989, S. 55, siehe auch die detaillierte Übersicht in: Caixun Nr. 74 (Mai 1988), S. 148.

60 Zhang Junhong, Taiwan gongshangren (Taiwanesische Unternehmer), S. 55 ff. mit einer Übersicht über die personellen Verknüpfungen, ebenda S. 58.

61 So wörtlich Liang Yonghuang in: Caixun Nr. 74 (Mai 1988), S. 115.

62 Siehe Zhongguo shibao vom 31.1.1992 und Ziyou shibao vom 5.3.1992.

63 Siehe den Parlamentsbericht in: Zhongguo shibao vom 24.2.1992.

64 Siehe die zusammenfassende Darstellung in Chen Shimeng u.a., Dang guo zibenzhuyi/KMT-State Capitalism, S. 205 ff. 
für Import- und Exportlizenzen oder überhöhte Wechselkurse verlangt, wobei der den offiziellen Kurswert übersteigende Betrag der Parteikasse zugeflossen sei. Das von der KMT im Jahre 1959 gegründete Finanzinstitut Zhonghua kaifa xintuo touzi gongsi hätte die Verwendung eines Großteiles der US-amerikanischen Hilfsgelder an sich gerissen und so von taiwanesischen Banken und privaten Untemehmen profitiert. In 1960 wurden in einem Aufsatz "Der Fiskus ist nicht die Parteikasse der KMT" direkte Zuwendungen aus dem Haushalt etwa für antikommunistische Propaganda und die Gründung von Parteizellen in allen Behörden und öffentlichen Unternehmen mit haushaltsmäßiger Verknüpfung gerügt.

Die Untemehmenspolitik der KMT, nämlich die Zusammenarbeit von Fiskus und Parteiunternehmen, wird bis heute fortgesetzt, etwa durch Gewährung von Vorzugskrediten ohne Sicherung an Parteiuntemehmen.65

Als in 1989 die KMT-Regierung entschied, in der Folgezeit gemäß Abschnitt 2 Bankengesetz Privatbanken zuzulassen, machte sich die KMT - mit ausdrücklicher Zustimmung des Finanzministers66 - sofort daran, zusammen mit anderen Unternehmen eine Parteibank zu gründen.67 Seit dem der Versicherungsmarkt geöffnet worden ist, bereitet die KMT den Aufbau eines Versicherungsunternehmens vor.68

Im Verständnis des Präsidenten des Verwaltungshofes Hao Bocun, also des Regierungschefs der KMT, sind die Parteiunternehmen der KMT schlicht Privatunternehmen.69 Aus Sicht der taiwanesischen Privatuntemehmer hingegen werden sie durch diese Wirtschaftsverfassung aus weiten Bereichen des taiwanesischen Binnenmarktes hinausgedrängt. Privilegierte Unternehmen des Staates und der KMT schränken auf diese Weise die gemäß

65 Chen Shimeng u.a., ebenda, S. 206 und das Jahr 1991 betreffend die Berichte in Minzhong ribao vom 22. und 23.3.1991.

66 Finanzminister Wang Jianxuan vertrat die Ansicht, daß jede Partei einen Antrag auf eine Bankgründung stellen könne. Parteien seien Organisationen des Volkes und könnten einen Antrag auf Zulassung einer Partei-Bank stellen, sofern er nur die gesetzlichen Voraussetzungen erfülle, siehe Zhonghua ribao vom 22.6.1990. Das Problem ist hier, daß die Gesetze einseitig von der KMT bestimmt worden sind und die KMT bis heute die Ausarbeitung eines Parteigesetzes ablehnt.

67 Siehe den Bericht in der Wirtschaftszeitung Gong shang shibao vom 20.9.1990. Der vorn Vorsitzenden der Finanzkornmission der KMT mit der Bankgründung beauftragte frühere Vorstandsvorsitzende der Taiwan Bank Xie Rendong sagte in einem Presseinterview ausdrücklich: "Ich denke, die KMT will finanziell selbstständig sein, neben dem unternehmerischen Mittel, Fimen zu betreiben, gibt es dann noch den Weg, eine Bank zu gründen, um die eigenen Unternehmen zu unterstützen", und auf die Frage, was diese Bank von anderen ebenfalls in Gründung befindlichen Privatbanken unterscheide, antwortete er:"Weil "die Partei" (diese Bank) betreibt, kann eine Kreditwürdigkeit leicht aufgebaut werden ..."(sic!), in: Shangye zhoubao/Business Weekly Nr. 109 (25.12.1989), S. 58.

68 Siehe die Berichte in den Tageszeitungen Zili zaobao vom 28.1.1992, Lianhe bao vom 16.2.1992 und 20.3.1992 und Ziyou shibao vom 23.3.1992.

69 Lianhe bao vom 20.3.1992. 
Art. 15 Verf geschützte Unternehmerfreiheit, insbesondere die Freiheit der Gründung eines Unternehmens und des Marktzutritts, erheblich ein. Für eine westliche Wirtschaftsverfassung ist die Entkoppelung von Politik und Wirtschaft als Kennzeichen angegeben und gefolgert worden:

"Für die Politik bedeutet das: Beschränkung ihres Einflusses. Diese Beschränkung läßt sich aber nur auf Dauer durchsetzen, wenn es gelingt, die Wirtschaft dauerhaft politisch zu neutralisieren. Politik muß also auf Hegemonie verzichten, darf aber auch nicht käuflich werden".70

Durch den Aufbau des Wirtschaftsimperiums der KMT hat die herrschende Flüchtlingselite der KMT in Taiwan aus Gründen ihres Machterhaltes es genau an dieser Beschränkung gegenüber der Wirtschaft fehlen lassen.

Darüber hinaus hat der Eingriff in die Unternehmerfreiheit durch privilegierte Parteiunternehmen noch eine weitere Folge, nämlich auf die innerparteiliche Demokratie. Betreffend die Parteienfinanzierung ist in der deutsche Verfassungslehre gewamt worden, daß "eine staatliche (Teil-)Finanzierung der Parteien die Gefahr einer Lockerung ihrer Orientierung an ihren Mitgliedern mit den bekannten Folgen einer Entpolitisierung und Personalisierung gesellschaftlicher Konflikte und damit der Vernachlässigung der ihnen in Art. 21 Abs. 1 Satz 1 zugewiesenen Funktion (erzeuge)".71 Mit der jahrzehntelangen Aufrechterhaltung des Kriegsrechtes und Ablehnung von Neuwahlen wurde die taiwanesische Bevölkerung(smehrheit) von politischen Entscheidungen ausgeschlossen. Mit dem Aufbau von Parteiuntemehmen machte sich die aus der vom Festland stammenden Flüchtlingselite bestehende Parteispitze der KMT zusätzlich von einem finanziellen Einfluß von taiwanesischen Parteimitgliedern unabhängig.

\section{Die Wirkungen dieser Einschränkungen}

Im Folgenden soll auf die Wirkungen dieser Einschränkungen der Untemehmerfreiheit eingegangen werden, wobei sich eine weitere Besonderheit der taiwanesischen Wirtschaft zeigt.

70 Roellecke, Der Staat 30 (1991), S. 391.

71 AK-GG-Preuß, 2. Aufl. 1989, Art. 21 Abs. 1, 3 Rdnr. 79 m.w.Nachw. So auch das jüngste Urteil des BVerfG zur Parteienfinanzierung, siehe Der Spiegel Nr. 16/1992, S. 35 ff. 


\subsection{Klein- und mittelständische Export-Unternehmen}

Die starke Konzentration von politischer und wirtschaftlicher Macht der KMT hat sich wie gezeigt in der Schaffung monopolartiger oder sonst privilegierter Unternehmen des Staates und der KMT ausgedrückt. Betreffend den Binnenmarkt ist noch zu berücksichtigen, daß die vom Festland geflohene Elite der KMT zu ihrer Herrschaftssicherung in Taiwan lokale, taiwanesische Interessengruppen, sofern sie der KMT arıgehören, mittels Gewährung örtlich begrenzter, wirtschaftlicher Monopolrechte, wie zum Beispiel Banken, Kreditgenossenschaften, Bauern- und Fischereiverbände und hier insbesondere deren Kreditabteilung sowie örtliche Verkehrsunternehmen, fördert. Nach einer Untersuchung gibt es in Taiwan 89 derartige örtliche Interessengruppen der KMT, wovon 81 Interessengruppen an diesen privilegierten, monopolartigen Wirtschaftsunternehmen beteiligt sind.72 Auch dies ist wiederum eine Einschränkung der Unternehmerfreiheit, nämlich der Freiheit des Marktzutritts.

Diese Einschränkungen der Unternehmerfreiheit haben dazu geführt, daß eine sehr große Zahl von Klein- und mittelständischen Unternehmem 73 sich im verbliebenen Bereich der Unternehmerfreiheit, dem Export, konzentriert und nach ausländischen Märkten strebt. Da der Export die Hauptantriebskraft des taiwanesischen Wirtschaftswachstums gewesen ist, dann kann der Beitrag der Klein- und mittelständischen Unternehmen zum taiwanesischen Wirtschaftswachstum nach Ansicht der modernen taiwanesischen Wirtschaftswissenschaft nicht hoch genug einschätzt werden. 74

In Taiwan gab es in 1987740.000 Klein- und mittelständische Unternehmen mit einem Anteil an der Gesamtzahl aller Unternehmen von 97,6\%.75 Betreffend den Gesamtexport hatten sie einen Anteil von über $60 \%$ und im Exportbereich der Güterproduktion sogar von

72 So Zhu Yunhan, Guazhan jingji yu weiquan zhengzhi tizhi (Das System aus oligopolisierter Wirtschaft und autoritärer Politik) in: Longduan yu boxue_(Monopolisierung und Ausbeutung), S. 151 unter Bezugnahme auf eine unter Federführung des Politikwissenschaftlers der Taiwan Universität Professor $\mathrm{Hu}$ Fo durchgeführte Studie.

73 Als Klein- und mittelständische Untemehmen gelten in Taiwan nach der Verwaltungsrichtlinie zur Betreuung von Klein- und mittelständischen Untemehmen (Zhong xiao qiye fudao zhunze) in der Fassung von 1982 z.B. im warenproduzierenden und verarbeiteten Gewerbe Untemehmen mit einem Eigenkapital unter 40.000.000 NT\$ und einem Gesamtvermögen unter 120.000.000 NT\$, im Bereich des Handels Untemehmen mit einem Umsatz von unter 40.000.000 NTS.

74 So ausdrücklich Liu Jintian/Chen Zhongrong, Zhong xiao qiye de shengcun yu fazhan (Überleben und Entwicklung der Klein- und mittelständischen Untemehmen), in: Quan guo minjian jingji huiyi shilu (Nationale private Wirtschaftskonferenz), Band 2, S. 91. Yow-shan Höfer, Die Außenhandelsverhandlungen zwischen Taiwan und den USA und ihre Auswirkungen auf die Klein- und Mitteluntemehmen in Taiwan, Hamburg 1991, S. 22 bezeichnet sie zu recht als "die Hauptträger der taiwanesischen Wirtschaft".

75 Liu Jintian/Chen Zhongrong, ebenda, Band 2, S. $91 \mathrm{f}$. 
bis zu $70 \%(!) .^{76}$ In der taiwanesischen Wirtschaftswissenschaft wird dabei folgendes hervorgehoben: Unter den oben genannten Bedingungen hätten sich die Klein- und mittelständischen Unternehmen die ausländischen Märkte als Ziel gesetzt, was zu einer Situation geführt habe, daß sehr viele kleine Unternehmen zu Exportuntemehmen geworden seien. Diese Situation erscheine taiwanesischen Landsleuten als schlicht natürlich und selbstverständlich. Verglichen aber mit den Erfahrungen anderer Staaten, und zwar egal ob es sich um industrialisierte Staaten, um neu industrialisierte Staaten, wie etwa Korea, oder um sich gerade entwickelnde Staaten, wie etwa Malaysia, handele, sei die Tatsache, daß die taiwanesischen Klein-und mittelständischen Unternehmen sich auf dem internationalen Markt einen Platz verschaffen konnten, ja Pioniere des taiwanesischen Exports geworden seien, eine ganz besondere Erscheinung. 77

Gegenüber regierungsoffiziellen und neoklassischen Darstellungen hat Menze $78 \mathrm{kritisch}$ angemerkt:

"Der größte Mythos ist dabei wohl die Behauptung, daß das 'taiwanesische Entwicklungsmodell' im wesentlichen dem Wirken von Liberalismus, Freihandel, freiem Unternehmertum und Marktkräften zu verdanken sei".

Menzel hat insoweit recht, als die Unternehmerfreiheit wie gezeigt, was den Binnenmarkt betrifft, erheblichen Beschränkungen unterliegt. Der Erfolg der taiwanesischen Wirtschaft ist andererseits aber gerade in dem - verbliebenen - Bereich der Untemehmerfreiheit erreicht worden, nämlich dem Export. Dort, wo sich die Unternehmerinitiative frei entfalten konnte, hat sie auch entscheidend zum Wirtschaftsaufschwung beigetragen. 79

76 Siehe die Angaben in Zhang Junhong, Taiwan gongshangren (Taiwanesische Unternehmer), S. 28 ff.; Zhou Tiancheng, Quanli bianchui de zhong xiao qiye (Klein- und mittelständische Unternehmen am Rande der politischen Macht) in: Longduan yu boxue - Weiquanzhuyi de zhengzhi jingji fenxi (Monopolisierung und Ausbeutung - Eine politisch-wirtschaftliche Analyse eines autoritären Regimes), hrsg. v. Taiwan yanjiu jijinhui (Forschungsstiftung Taiwan), Taipei, 1989, S. 109; Liu Jintian/Chen Zhongrong, Zhong xiao qiye de shengcun yu fazhan (Überleben und Entwicklung der Klein- und mittelständischen Unternehmen), in: Quan guo minjian jingji huiyi shilu (Nationale private Wirtschaftskonferenz), Band 2, S. 92.

77 Zhou Tiancheng, Quanli bianchui de zhong xiao qiye (Klein- und mittelständische Unternehmen am Rande der politischen Macht) in: Longduan yu boxue (Monopolisierung und Ausbeutung), S. 100.

78 Ulrich Menzel, In der Nachfolge Europas - Autozentrierte Entwicklung in den ostasiatischen Schwellenländem Südkorea und Taiwan, München 1985, S. 154.

79 Weitere Faktoren des taiwanesischen Wirtschaftsaufschwunges sind der infrastrukturelle Aufbau der Japaner während der Kolonialzeit, die ernorme Wirtschaftshilfe der Vereinigten Staaten und die Existenz einer entsprechenden Nachfrage auf dem Weltmarkt. 
Diese besondere Erscheinung ist auch die Folge anderer wirtschaftsrechtlicher Maßnahmen: Aufgrund der Erfahrungen mit der Hyperinflation auf dem Festland legte die KMTRegierung bei Schaffung des Neuen Taiwan Dollars (NT\$) einen hohen, wie sich bald zeigte überhöhten Wechselkurs fest. Die Beseitigung dieses Problems, welches den Export behinderte und den Import begünstigte, war das Hauptanliegen der Exportsubstitutionspolitik Anfang der sechziger Jahre. Man hatte damals zwei Möglichkeiten:80 Einmal hätte man sich konsequent öffnen können, den Wechselkurs anpassen, die Einschränkungen der Unternehmerfreiheit abbauen sowie die Zölle insbesondere für Rohstoffe und Maschinen senken können und den taiwanesischen Unternehmer, gestützt auf den Vorteil billiger Arbeitskräfte, auf dem internationalen Markt konkurrieren lassen. Dies tat man nicht, statt dessen hielt die Regierung an der bürokratischen Kontrolle und an den Zöllen grundsätzlich fest und beseitigte nur Hindernisse für den Exportsektor der Wirtschaft. Damals wurde nicht etwa die frühere Importsubstituierungspolitik grundlegend reformiert, sondern umgekehrt dem schon bestehenden, äußerst komplizierten, bürokratischen System nur Ausnahmeregeln für den Export hinzugefügt, was der Hauptinhalt des Investitionsförderungsgesetzes war.81 Statt die in der Verfassung garantierte Unternehmer- beziehungsweise Wirtschaftsfreiheit umfassend zu gewähren, wurde an dem System der im Binnenmarkt privilegierten Staats- und Parteiunternehmen und der wirtschafts- und verwaltungsrechtlichen Kontrolle der Wirtschaft festgehalten und nur Ausnahmenormen für den Export hinzugefügt.

Bereits in den fünfziger Jahren hatte die liberale Wirtschaftskritik in Taiwan kritisch gef ragt, wie sich unter diesen Bedingungen Unternehmen normal entwickeln sollen. 82 Sie haben sich, wie gezeigt, auch nicht normal entwickelt, was auch zu den gegenwärtigen Problemen geführt hat. Die ungesunde Finanzierung taiwanesischer Unternehmen sei dabei als besonderes Problem hier hervorgehoben.

Anfangs waren in Taiwan finanzielle Mittel knapp, weil es keine Devisen gab. Mit dem beginnenden Wirtschaftswachstum wurde gespart und man sollte annehmen, daß nun Banken Investitionsvorhaben von Unternehmern finanzierten. Das Bankengesetz sieht auch ausdrücklich die Gründung von Privatbanken nach staatlicher Genehmigung vor. Tatsächlich aber weigerte sich die KMT-Regierung jahrzehntelang entsprechend dem Bankengesetz Privatbanken zuzulassen, der Währungs-und Finanzsektor ist jahrzehntelang von staatlichen Banken beherrscht worden. In diesem durch Protektion geschützten Bereich ohne

80 Zum Folgenden siehe Zhou Tiancheng, Quanli bianchui de zhong xiao qiye (Klein- und mittelständische Unternehmen am Rande der politischen Macht) in: Longduan yu boxue (Monopolisierung und Ausbeutung), S. 105.

81 Die Entwicklung wird beschrieben von Ti-ying Cheng, Statute for Encouragement of Investment, Caishui yanjiu/Public Finance Review, Vol. 21 No.3 (Mai 1989), S. 1-20.

82 Siehe die Analyse Women de jingji (Unsere Wirtschaft) in: Ziyou zhongguo (Freies China) Band 17 Heft 6 (16.9.1957), S. 163 ff. 
Konkurrenz verlor der Finanzsektor seine Vermittlungsfunktion, mit der Folge eines gespaltenen Finanzmarktes, in welchem offizielle Finanzinstitute und unorganisierte "Untergrund-Finanzaktivitäten" (Dixia jinrong huodong) nebeneinander existieren. Nach taiwanesischen Untersuchungen beziehen inländische Unternehmen nur etwa $60 \%$ ihrer Finanzmittel vom offiziellen Finanzbereich, wovon etwa ein Drittel den Klein- und mittelständischen Untemehmen zufließt. Klein- und mittelständische Untemehmen können ihren Finanzbedarf nur zu etwa einem Drittel im offiziellen Finanzmarkt decken. 83

Hierbei wird auch die ganze Problematik der Sondergesetze in der Wirtschaftsverfassung Taiwans deutlich: Aufgrund von $\S 18$ des "Gesetzes der nationalen Generalmobilmachung" erließ die KMT-Regierung die "Verordnung zur Unterdrückung von Untergrund-Geldhäusem".84 Andererseits waren taiwanesische Unternehmer aufgrund der rechtlich bedenklichen und marktfeindlichen Finanzpolitik derselben KMT-Regierung gezwungen, sich Kredite zu überhöhten Zinsen aus dem Untergnund zu besorgen. Taiwanesische Sparer sind ihrerseits gezwungen gewesen, ihr Geld im Untergrund-Finanzmarkt anzulegen, da offizielle Banken aufgrund ihrer wettbewerbsausschaltenden Privilegien nicht gezwungen waren, attraktive Anlagemöglichkeiten anzubieten.

Nachdem die den Export fördernde Währungspolitik, das heißt die bewußte Unterbewertung des Neuen Taiwan Dollars, aufgrund ausländischen Drucks nicht mehr fortgesetzt werden konnte und der Wechselkurs gestiegen ist, sind die Klein- und mittelständischen Unternehmen in existentielle Bedrängnis geraten. Der Wegfall des Währungsvorteils verursachte nach Angaben der jetzigen Vorsitzenden des Council for Economic Planning and Development Guo Wanrong ein bedenkliches Absinken privater Investitionen, außer bemerkenswerterweise im Bereich der illegalen Schattenwirtschaft, auf die unten noch einzugehen sein wird.85 Die Schwäche der bisherigen Wirtschaftsverfassung ist nunmehr zutage getreten: Die Klein- und mittelständischen Unternehmen werden aus Taiwan gedrängt und müssen im südostasiatischen Ausland und in China investieren, mit der Folge unzureichender privater Investitionen in Taiwan selbst. 86

Zhou Tiancheng, Quanli bianchui de zhong xiao qiye (Klein- und mittelständische Unternehmen am Rande der politischen Macht) in: Longduan yu boxue (Monopolisierung und Ausbeutung), S. 112.

84 Qudi dixia qianzhuang banfa vom 21.5.1952.

85 Siehe den Bericht in Lianhe bao vom 29.3.1991.

86 Nach Angaben des Wirtschaftsministeriums steht Taiwan mit seinen Auslandsinvestitionen in Höhe von 19 Milliarden US\$ nunmehr weltweit an neunter Stelle, siehe The Free China Journal vom 10.4.1992. 


\subsection{Illegale Schattenwirtschaft}

Ein weiterer gravierender Aspekt dieser Wirtschaftsverfassung ist die weit verbreitete, illegale Schattenwirtschaft sowie deren steigende Tendenz.

Nach in der Presse im Mai 1988 veröffentlichten Angaben des Wirtschaftsministeriums hätte es damals über 17.000 illegale Fabriken gegeben87, im Juli 1988 wurde eine Zahl von über 21.000 veröffentlicht.88 In 1985 und 1987 wurden Kampagnen der großzügigen Registrierung zur Legalisierung durchgeführt, dennoch gäbe es noch knapp 20.000 illegale Fabriken. 89 Eine Hinweis-Kampagne der Regierung blieb erfolglos, worauf der Präsident des Verwaltungshofes Hao Bocun im Juli 1990 mit kraftvollen Worten energische Maßnahmen androhte. Zwei Monate später stellte eine Provinzbehörde entgegen den bisherigen Angaben der örtlichen Kreisbehörden sogar knapp 70.000 illegale Fabriken fest.90 In Taiwan gab es danach im Jahre 1989186.466 legale Fabriken und daneben immerhin 69.847 illegale Fabriken, davon würden von den Behörden 118 Fälle, also 0,17 \%, jährlich bearbeitet werden. 91

Im übrigen gibt es 234.000 Einzelhändler, die ohne Lizenz, also illegal wirtschaften.92 Über $70 \%$ der gewerblichen Fischzucht ist illegal, weniger als $25 \%$ der genutzten Seeoberfläche werden angemeldet.93 Von 120 Freizeitparks im Grünen sind 70 illegal.94 Die 748 KTV-Unternehmen des Vergnügungsgewerbes, bei denen die Gäste zu vertonten Videos selbst singen können, sind alle illegal; allein in Taipei gibt es 2200 illegale und nur 1800 legale Vergnügungslokalitäten.95 Und schließlich sind von 21 Golfplätzen nur 2 legal.96

Nach einer Statistik der zuständigen Provinzbehörde von 1958 gab es in Taiwan 23.097 illegale Gebäude, bis 1991 war die Zahl auf 104.323 illegale Gebäude angestiegen.97 Ein Fünf-Jahres-Plan zum Abriß illegaler Bauten setzte Kosten von über 1 Milliarde NT\$ an.98

87 Gong shang shibao vom 5.5.1988.

88 Zhonghua ribao vom 5.7.1988.

89 Jingji ribao vom 11.12.1989.

90 Zhongguo shibao vom 3.9.1990.

91 Statistik nach der Arbeitsbehörde der Provinz in: Lianhe baovom 1.9.1990.

92 China Post vom 8.9.1989.

93 Lianhe bao 10.4.1991.

94 Zhongguo shibao vom 4.3.1992.

95 Lianhe bao vom 17.9.1990.

96 Siehe die Liste in: Zhong guo shibao vom 26.2.1992.

97 Lianhe bao vom 27.3.1991.

98 Zhongguo shibao vom 4.12.1991. 
Eingangs ist darauf hingewiesen worden, $\mathrm{da} \beta$ in einem marktwirtschaftlichen System die Trennung von Staat und Wirtschaft unter anderem auch die Kontrolle der Wirtschaft durch den Staat erleichtern und damit die Wahrscheinlichkeit erhöhen soll, daß den allgemeinen, nichtwirtschaftlichen Interessen Rechnung getragen wird. Die Duldung der Illegalität vor allem in den Bereichen des Schutzes allgemeiner öffentlicher Interessen (Gesundheits,Natur-, Wasser- und Landschaftsschutz, Bau- und Verkehrssicherheit und so weiter) hat hier die Funktion eines ausgleichenden Sicherheitsventils gegenüber den oben beschriebenen Einschränkungen der Unternehmerfreiheit. Diese Privatunternehmer besänftigende und damit auch die Herrschaft der KMT sichemde Funktion führt nun allerdings dazu, daß die Regierung gegenüber den Bürgern ihre Schutzfunktion nicht im notwendigen Maße wahrnimmt.

Betreffend die Unternehmer ist noch folgender Unterschied zur Lage in Deutschland zu berücksichtigen. In der deutschen Verfassungslehre ist auf einen eingetretenen Bedeutungsverlust des Eigentums als Grundlage privater Existenzsicherung und individueller Lebensgestaltung hingewiesen worden. Grundlage individueller Existenzsicherung und Lebensgestaltung sei überwiegend nicht mehr das private Eigentum im überkommenen Sinne des bürgerlichen Rechts, sondern die eigene Arbeit und die Teilhabe an den Leistungen staatlicher Daseinsvorsorge und Fürsorge.99 Dies ist natürlich anders in einem Land ohne eine vergleichbare staatliche Fürsorge und einer Mehrheit von Klein- und mittelständischen Unternehmen. Hier ist das Privateigentum, der Familienbetrieb tatsächlich noch die Existenzgrundlage weiter Kreise der Bevölkerung, weswegen die politisch motivierten Eingriffe in das Privateigentum und Beschränkungen der Unternehmerfreiheit besonders schwer wirken.

In wirtschaftlicher Sicht ist die Illegalität eine Notwendigkeit der kleinen Unternehmer, um durch radikale Kostensenkung in dem harten internationalen Wettbewerb überhaupt bestehen zu können. Die taiwanesischen Klein- und mittelständischen Unternehmer haben in dem ihnen verbliebenen Bereich der Unternehmerfreiheit, vor allem dem Export, überhaupt nur eine Chance, wenn sie ihre Kosten erheblich senken und extrem günstige Preise anbieten. In diesem Zusammenhang ist es zu sehen, wenn sie neue westliche Technologie und in ausländischen Märkten gerade neu entwickelte Produkte imitieren. 100 Beipielhaft mag hier der vorübergehende Zusammenbruch der Wirtschaftsverhandlungen zwischen den Vereinigten Staaten und Taiwan betreffend den Schutz geistigen Eigentums und die

99 Konrad Hesse, Grundzüge des Verfassungsrechts der Bundesrepublik Deutschland, Heidelberg, 17. Aufl. 1990, S. 174 Rdnr. 443.

100 Eine seltene, punktuelle Andeutung dieses Bereiches der illegalen Schattenwirtschaft in: Liu Jintian/Chen Zhongrong, Zhong xiao qiye de shengcun yu fazhan (Uberleben und Entwicklung der Klein- und mittelständischen Unternehmen), in: Quan guo minjian jingji huiyi shilu (Nationale private Wirtschaftskonferenz), Band 2, S. 91. 
anschließende, zeitweise Sanktion genannt werden, Taiwan auf die besondere § 301-Beobachtungsliste zu setzen. 101

Aus kritischer Sicht wurde schließlich noch ein politischer Aspekt der Duldung der llegalität betont: Die vom chinesischen Festland stammende, herrschende Flüchtlingselite der KMT dulde zur Sicherung der Loyalität örtlicher, taiwanesischer Interessensgruppen der KMT es, daß diese sich mittels der öffentlichen Macht lokaler Regierungen wirtschaftliche Vorteile verschaffen. Die lokalen Interessengruppen der KMT könnten im übrigen direkt oder indirekt die Polizei, die Justiz, die Verwaltung u.s.w. beeinflussen, illegale Wirtschaftsunternehmen großzügig zu behandeln. 102

Im Hinblick auf die Wirtschaftsverfassung und ihre problematischen Folgen wird nun zu fragen sein, wie die liberale Opposition in Taiwan dazu Stellung genommen hat.

\section{Die Forderungen der liberalen Opposition}

Die oben beschriebene Wirtschaftspolitik der KMT und die damit verbundene Ausformung der Wirtschaftsverfassung ist in Taiwan von Anfang an von Liberalen stark kritisiert worden.

Insbesondere aufgrund der Verstaatlichung der japanischen Unternehmen durch die chinesische Nationalregierung und der Besetzung der Führungspositionen in Wirtschaft und Verwaltung mit Festlandschinesen, die in den Augen der Taiwanesen als Fortsetzung der Kolonialpolitik, als Fortsetzung der Fremdbeherrschung ihrer Wirtschaft erschien, kam es zu Auseinandersetzungen mit Festlandschinesen. Die Truppen der Nationalregierung nahmen diesen Protest zum Anlaß, um eine in der taiwanesischen Geschichte einmalige Barbarei unter den Taiwanesen anzurichten und damals zwischen 18.000 und 28.000 Taiwanesen zu ermorden.103 Während dieses "Vorfalles vom 28. Februar (1947)" legte die ad hoc zusammengetretene, taiwanesische "Kommission zur Behandlung des Vorfalles vom 28.

101 Siehe The Free China Joumal vom 14. und 17.4.1992. $\mathrm{Zu}$ den Verhandlungen allgemein siehe Yow-shan Höfer, Die Außenhandelsverhandlungen zwischen Taiwan und den USA und ihre Auswirkungen auf die Klein- und Mitteluntemehmen in Taiwan, Hamburg 1991.

102 Zhu Yunhan, Guazhan jingji yu weiquan zhengzhi tizhi (Das System aus oligopolisierter Wirtschaft und autoritärer Politik) in: Longduan yu boxue (Monopolisierung und Ausbeutung), S. $151 \mathrm{f}$. Detaillierte, nachprüfbare Tatsachen können und werden vom Autor naturgemäß nicht angegeben. Ich gebe diese Meinung hier vorläufig als eine Stimme in der neuen, noch in der Entwicklung begriffenen, kritischen Wissenschaft in Taiwan wieder.

103 Siehe Anlage 5 des offiziellen Abschlußberichtes ("Er erba shijian" yan jiu baogao), der Regierungskommission zur Untersuchung des Vorfalles vom 28. Februar (1947) (Xingzhengyuan yan jiu "er erba shijian" xiao zu), der im Februar 1992 vorgelegt worden ist. Hierbei ist zu berücksichtigen, daß die Bevölkenung Taiwans nur sechs Millionen Menschen zählte. 
Februar" ein Programm über zu ergreifende Maßnahmen vor. Die darin enthaltenen, die Wirtschaft betreffenden Forderungen sind die erste Kritik an der Wirtschaftspolitik der KMT in Taiwan:

"16. Die Leitungspositionen aller öffentlichen Unternehmen werden von (taiwanesischen) Provinzangehörigen besetzt.

17. Einrichtung einer vom Volk gewählten Kommission zur Ưberwachung öffentlicher Unternehmen, die Vollmacht zur Regelung des japanischen Vermögens ist der (taiwanesischen) Provinzregierung zu übertragen, in allen (von den Japanem) übernommenen Fabriken und Bergwerken sind Kommissionen zur Unternehmensführung einzurichten, über die Hälfte der Kommissionsmitglieder müssen (taiwanesische) Provinzangehörige sein.

18. Abschaffung des Verkaufsamtes (Zhuanmaiju), Güter des täglichen Bedarfs sind einem Rationierungssystem zu unterwerfen.

19. Abschaffung des Handelsamtes (Maoyiju)".104

Die Forderungen verhallten unbeachtet. Nach diesem Massaker unterdrückte die KMTRegierung politischen Dissent, in den fünfziger Jahren schwergewichtig mit der Todesstrafe, eine Periode die heute in Taiwan mit "Periode des weißen Terrors" (Baise kongbu shiqi) bezeichnet wird. In den sechziger Jahren ging sie schwergewichtig zur Verhängung lebenslanger Freiheitsstrafen über.105

Die ersten zehn Jahre der Diktatur von Chiang Kai-shek auf Taiwan griff der berühmte Professor der Taiwan Universität Yin Haiguang im Jahre 1959 mit den Worten an:

"Die tatsächliche Methode der Ein-Parteien-Diktatur ist es, Taiwan als das Parteivermögen einer Partei zu betrachten, ist es unter dem Namen 'Staat' und gestützt auf die Organe der Regierung die auf vielen Ebenen, auf der politischen, der militärischen, der

104 "Chuli da gang" der Kommission "Er erba shijian chuli weiyuanhui", welche den ersten organisierten, taiwanesischen Protest bildete, unter anderem abgedruckt in: Er erba shijian ziliao ji (Materialsammlung zum Vorfall vom 28. Februar), zusammengestellt von Deng Kongzhao, Taipei 1991, S. 271 ff. Diese oppositionelle, taiwanesische Kommission wurde noch im März 1947 innerhalb weniger Tage durch das Militär gewaltsam, das heißt durch Tötung, Verschwindenlassen oder Verhaftung, aufgelöst. Siehe dazu Li Xiaofeng, Taiwan minzhu yundong sishi nian (40 Jahre taiwanesische Demokratiebewegung), Taipei 3. Aufl. 1989, S. 50 f.

105 Siehe die Aufstellungen in LuXiulian, Chong shen meilidao (Emeute Untersuchung des Vorfalles des Formosa-Magazins), Taipei 1991, S. 19 ff. 
wirtschaftlichen, der pädagogischen und der gesellschaftlichen Ebene durchgeführte Ausrichtung auf die Partei".106

Demgegenüber wurde von einem liberalen Verständnis der Zusammenhang von Demolratie und Marktwirtschaft betont:

"Eine politische Demokratie braucht eine freie Wirtschaft als Grundlage. Staatliche Unternehmen im Bereich der produktiven Wirtschaft können nur in einer Situation der Ressourcenknappheit zur Vermeidung privater Monopolisierung erforderlich sein; über diese Grenze hinaus bedeutet eine Ausweitung staatlicher Unternehmen eine entsprechende Schwächung der Demokratie. Der Grund liegt einfach darin, daß wenn der Geldbeutel der Regierung vom Volk (vermittels seiner Vertreter) in den Händen gehalten wird, das Volk erst die Regierung kontrollieren und sie dazu bringen kann, nicht ihre Macht zu mißbrauchen. Wenn umgekehrt das wirtschaftliche Leben des Volkes in der Hand der Regierung liegt, dann kann diese Regierung einen autoritären Weg beschreiten oder es ist bereits eine autoritäre Regierung".107

Staatsuntermehmen gediehen im übrigen nur unter einer protektionistischen Politik. Man sollte überlegen, diese protektionistische Politik auch betreffend die Industrie abzuschaffen.108 Diese erforderlichen Reformen müßten auf ein eindeutiges Ziel gerichtet sein: die Entwicklung freier Wirtschaftsunternehmen. Es wurde verlangt, die Verwaltungsaufsicht über Unternehmen der Industrie und des Handels vollständig und rasch und die Verwaltungsaufsicht über Währung und Finanzen schrittweise abzuschaffen.109

"Wir meinen, daß die heute (wirtschaftlich) rückständigen Staaten zum Zwecke einer normalen wirtschaftlichen Entwicklung die Verwaltungsaufsicht über die Wirtschaft auf ein Mindestmaß reduzieren müssen, eine schlechte Kontrolle ist schlechter als keine Kontrolle". 110

Die Wirtschaft genauso wie die Politik, beide benötigten einen demokratischen Geist, die Regierung habe insbesondere betreffend die Klein- und mittelständischen Unternehmen

106 Yin Haiguang, Ziyou zhongguo zhi lu (Der Weg des freien China) in: Yin Haiguang quanji (Sämtliche Werke von Yin Haiguang) zusammengestellt von Lin Zhenghong, Taipei, 1990, Band 12 Zhengzhi yu shehui (Politik und Gesellschaft), S. 771 ff. (772).

107 Minying shiye de shiming (Die Mission der Privatuntemehmen), Kommentar in: Ziyou zhongguo (Freies China) Band 12 Heft 8 (16.4.1955), S. 251.

108 Ebenda S. 251.

109 So in der Wirtschaftsanalyse Women de jingji (Unsere Wirtschaft) in: Ziyou zhongguo (Freies China) Band 17 Heft 6 (16.9.1957), S. 167.

110 Chen Shirui, Lun zhengzhi de ganshi jingji (Über die Einmischung der Politik in die Wirtschaft), in: Ziyou zhongguo (Freies China) Band 18 Heft 3 (1.2.1958) S. 88. 
konkrete wirtschaftliche Hilfsmaßnahmen zu ergreifen. Alle größeren Industrieuntemehmen im freien China seien Staatsbetriebe. Weil nach der Beendigung des Kolonialismus in Taiwan alle Kräfte auf die staatlichen Unternehmen konzentriert worden seien, hätte die Regierung die privaten Klein- und mittelständischen Unternehmen nicht genügend beachtet. 111

Im übrigen setzte man sich intensiv mit dem Wirtschaftsaufschwung von Westdeutschland auseinander.112 Professor Yin Haiguang zog damals folgenden Vergleich:

"Wenn wir 'an einer anti-kommunistischen Hoffnung festhalten' wollen, dann müssen wir vom freien Westdeutschland lemen und mit dem freien Westdeutschland denselben Weg beschreiten. Seit zehn Jahren stehen sich das freie Westdeutschland und das rote Ostdeutschland gespannt gegenüber. Aber das freie Westdeutschland unterdrückt nicht wie das 'Führungszentrum' bei uns, gestützt (auf die Parole) 'Wider den Kommunismus, Widerstand gegen Rußland' Freiheit und Demokratie ... Noch viel weniger zerstört das freie Westdeutschland unter dem Namen des Antikommunismus die Lebenskraft der Gesellschaft. Genau umgekehrt, das freie Westdeutschland schreitet unter der Verantwortung von Adenauer und Erhard rasch auf dem Weg der Wiederbelebung voran".113

Schließlich verwies er noch auf über 3.000.000 Ostdeutsche, die nach Westdeutschland gekommen wären, ein Ergebnis, das keine Propaganda erreichen könne. Er führte es auf folgende drei Punkte zurück, die er damit auch für Taiwan forderte: politische Demokratie, wirtschaftliche Freiheit und unabhängige Wissenschaften.114

In den siebziger Jahren forderte der Senior der taiwanesischen, parlamentarischen Opposition Kang Ningxiang unter dem Titel "Wenn die Regierung ein Unternehmen wäre" die

111 Qu Jingzhou, Zhong xiao gongye jinrong zhi zhongyaoxing (Die Wichtigkeit der Finanzen der Klein- und mittelständischen Industrie), Ziyou zhongguo (Freies China) Band 8, Heft 2 (16.1.1953), S. 59.

112 Siehe beispielsweise $Y u$ Jian, Lun xide jingji de fuxing (Über die Wiederbelebung der Wirtschaft Westdeutschlands) in: Ziyou zhongguo (Freies China) Band 17 Heft 1 (1.7.1957), S. 11-15 und Band 17 Heft 2 (16.7.1957) S. 48-49.

113 Yin Haiguang, Ziyou zhongguo zhi lu (Der Weg des freien China) in: Quanji (Werke) Band 12 Zhengzhi y u shehui (Politik und Gesellschaft), S. $771 \mathrm{ff}$. (777 f.).

114 Prof. Yin Haiguan verstand sich nach eigenen Worten als ein Liberaler (Ziyouzhuryizhe), weswegen er auch in den Jahren 1953 und 1954 F.A.Hayek, The Road to Serfdom, ins Chinesische übersetzte, enthalten in: Yin Haiguang quanji (Sämtliche Werke von Yin Haiguang) zusammengestellt von Lin Zhenghong, Taipei, 1990, Band 6 Dao nuyi zhi lu. Er mußte schließlich sein liberales Eintreten mit dem Verlust seines Arbeitsplatzes bezahlen. Sein liberaler Gesinnungsgenosse Lei Zhen, der 1960 eine Oppositionspartei, die Demokratische Partei Chinas (Zhongguo minzhu dang), mitgründen wollte, wurde mit zehnjährigem Freiheitsentzug bestraft. 
Verwirklichung der verfassungsmäßigen Demokratie: Demokratische Staaten seien wie ein Unternehmen, das Volk sei dabei der Anteilseigner. Die Regierung sei lediglich die vom Anteilseigner gegen Bezahlung angestellten Manager, Buchhalter, Angestellten und so weiter. Die KMT-Regierung hingegen denke nicht so. Er forderte, so wie in der Wirtschaft der Anteilseigner solle das Volk selbst das Verhalten der vertraglich Angestellten überprüfen und entscheiden können, wer mit einer Lohnerhöhung zu fördern sei oder wer zu entlassen sei. 115

Die letzte große Verhaftungswelle wurde Ende 1979 von der Zentralen Militärkommandantur Taiwan (Taiwan jingbei zongsilingbu) nach dem dem "Vorfall des Formosa-Magazins" (Meilidao shijian), auch als "Kaoshiunger Vorfall" (Gaoxiong shijian) durchgeführt.116 Im Hinblick auf das jahrzehntelange Kriegsrechtsregime der KMT in Taiwan standen bei der taiwanesischen Opposition Forderungen nach Demokratie und einem verfaßten Rechtsstaat im Vordergrund. Erst Ende der achtziger Jahre - nach Aufhebung des Kriegsrechts -begann man sich kritisch mit Fragen der Wirtschaftsverfassung und hierbei insbesondere der Beschränkungen der Unternehmerfreiheit auseinanderzusetzen. 117

Dabei tauchte der Zusammenhang von Beschränkungen von wirtschaftlichen Freiheitsrechten zu autoriärer Herrschaftssicherung immer wieder in den Vordergrund. In der ganzen Welt gäbe keine politische Partei in einem demokratischen Staat, der derart große Parteiunternehmen gehören.118 Die KMT festige mittels der Überschüsse der Unternehmen des Staates und der Partei ihre politische Übermacht und verhindere so eine Entwicklung

115 Kang Ningxiang, Jaru zhengfu xiang qiye gongsi (Wenn die Regierung ein Unternehmen wäre), in: Zhao Jiawen/Chen $J u$ (Hrsg.), Dangwai wenxuan (Ausgewählte Texte der außerparteilichen Opposition), Taipei 1979, S. $20 \mathrm{ff}$.

116 Auf einer von der Opposition am 10. Dezember 1979 veranstalteten, nicht genehmigten Demonstration für die Einhaltung der Menschenrechte in Taiwan kam es zu gewaltätigen Auseinandersetzungen. Betreffend die Vorgänge während der Demonstration und darüber, wer mit den Gewaltätigkeiten begann, gibt es widersprüchliche Darstellungen, auf die hier nicht im Einzelnen eingegangen werden kann. Die Aktivistin für Freiheitsrechte und Frauenrechte Lu Xiulian, die auch zu den Verhafteten gehörte, hat nun die erste, umfassende Studie zu diesem Vorfall und seiner Vorgeschichte vorgelegt: dieselbe, Chong shen meilidao (Emeute Untersuchung des "Vorfalles des Formosa-Magazins", Taipei, 1991.

117 Hervorzuheben ist die Studie von Peng Baixian, Taiwan jingji tizhi de benzhi -kongzhi jingji (Die Qualität des Wirtschaftssystems Taiwans - eine verwaltete Wirtschaft), in: 1988 Taiwan gonggong zhengce yantaohui lunwenji (1988 Symposium über die öffentliche Politik in Taiwan - Aufsatzsammlung), hrsg.v. Beimei taiwanren jiaoshou xiehui(Vereinigung taiwanesischer Professoren in Nordamerika) und Taiwan renquan cujinhui(Taiwanesische Gesellschaft zur Förderung der Menschenrechte), Taipei 1989, S. 17-47.

118 Zhang Junhong, Taiwan gongshangren (Taiwanesische Unternehmer), S. 60. 
einer demokratischen Politik. ${ }^{119}$ In einem autoritären politischen System seien oligopolisierte oder monopolisierte Wirtschaftssektoren nicht nur natürlich entstandene Wirtschaftsergebnisse des Marktes, in einem derartigen System würden Politiker zusätzlich die Zwangsmacht des Staatsapparates benutzen, um durch Einschränkung des Wettbewerbes privilegierte Oligopole oder Monopole schaffen, um so neue, nach politischen Gesichtspunkten verteilbare, wirtschaftliche Renten zu schaffen. 120 Im Mai 1988 stellte der Abgeordnete der oppositionellen Demokratischen Fortschrittspartei (DFP) Yu Zhengxian in dem Wirtschaftsmagazin Caixun die bis heute unbeantwortet gebliebenen Fragen:

"Wieviele Unternehmen betreibt die KMT tatsächlich mittels ihrer Fraktionen und Privilegien? Und wenn die KMT-Regierung zur Zeit die Rückkehr zur Verfassung proklamiert, wie will die KMT auf diese ihre privilegierten Unternehmen verzichten und mit den anderen Parteien in einen gerechten Wettbewerb treten?"121

Und schließlich wandte man sich gegen wirtschaftsrechtliche Sanktionen gegen politisch Oppositionelle, nämlich daß diese neben einer strafrechtlichen Verurteilung noch mit einem anschließenden Berufsverbot verfolgt werden, etwa Prokuristen in einem Wirtschaftsunternehmen nach $\S 30$ Gesellschaftsgesetz (Gongsi fa), Ärzte nach § 5 Arztgesetz (Yishi fa), Tierärzte nach § 3 Tierarztgesetz (Shouyishi fa), Hebammen nach § 6 Ziff.1 Hebammengesetz (Zhuchanshi fa), Architekten nach $\$ 4$ Architektengesetz (Jianzhushi fa) und so weiter. Dagegen wandte man sich unter ausführlicher Darlegung des Apotheken-Urteils des Bundesverfassungsgerichtes und der dort entwickelten Stufentheorie.122

Von einem traditionellen, ausdrücklich auf Adam Smith gestützten Verständnis aus fordert die liberale, taiwanesische Wirtschaftswissenschaft als vorrangiges Ziel die Verstaatlichung der Parteiunternehmen und dann deren Privatisierung. 123 Vor dem Hintergrund der Aktivitäten der KMT, ihren unternehmerischen Bereich noch auszubauen, veröffentlichte die

119 Zhang Qingxi, Gongying yu dangying shiye de duzhan(Die Monopolisierung durch öffentliche Unternehmen und Unternehmen der Partei), in: Quan guo minjian jingji huiyi shilu(Nationale private Wirtschaftskonferenz), Band 2, S. $38 \mathrm{f}$.

120 So Zhu Yunhan, Guazhan jingji yu weiquan zhengzhi tizhi (Das System aus oligopolisierter Wirtschaft und autoritärer Politik) in: Longduan yu boxue (Monopolisierung und Ausbeutung), S. 139.

121 Yu Zhengxian in: Caixun Nr. 74 (Mai 1988), S. 116.

122 Qi Ye, Zhengzhifan ye yao shenghuo! - Zhengzhifan zhiye ziyou jibenquan ying shou baozhang ( Politische Straftäter wollen auch leben! Das Grundrecht der Berufsfreiheit von politischen Straftätern muß garantiert werden), in: Shoudu zaobao vom 27.8.1989. - Anfang März 1991 versprach der Präsident des Verwaltungshofes $\mathrm{Hao}$ bei der anstehenden Überarbeitung dieser und weiterer Gesetze, diese Beschränkungen zu streichen, Lianhe bao vom 1.3.1991. Im April 1992 ist ein entsprechender Passus aus dem Gesetz für Emährungsberater (Yingyangshifa) gestrichen worden, Zili zaobao vom 22.4.1992.

123 Chen Shimeng u.a., Dang guo zibenzhuyi/KMT-State Capitalism, S. 204 ff. 
Wirtschaftswissenschaftliche Gesellschaft Chinas die besondere Erklärung "Wider das Eindringen politischer Parteien in die Wirtschaft" vom 16.12.1990.

Aus Sicht der politischen Opposition forderte der jetzige Abgeordnete der Nationalversammlung und Mitglied der DFP Zhang Junhong, daß wenn man den Investitionswillen taiwanesischer Ünternehmer stärken wolle, man dann gleichfalls auch deren Probleme lösen und von der KMT verlangen müsse, ihr riesiges und monopolartiges Wirtschaftsvermögen dem Volk zurückzugewähren und eine Demokratie aufzubauen.124 Es sei ein äußerst ungerechter, wirtschaftlicher Wettbewerb, bei dem private Unternehmungen auf den Bereich der Produktion von Konsumgütern und den Export beschränkt würden. 125

Im August 1991 haben oppositionelle, taiwanesische Kräfte einen "Entwurf der Verfassung der Republik Taiwan" ausgearbeitet. $126 \mathrm{In}$ ihm wird das Eigentum wie folgt geregelt:

"Art. 22: Das Vermögensrecht wird garantiert, sein Inhalt und seine Schranken werden durch Gesetz bestimmt.

Der Gebrauch des Vermögens hat von gesellschaftlicher Verantwortung getragen zu sein. Verteilung, Erwerb und Veräußerung sowie Verwendung des Vermögens haben die öffentlichen Interessen der Gesellschaft zu berücksichtigen, um Spekulation und Monopolisierung sowie eine Gefährdung öffentlicher Interessen der Gesellschaft zu verhindern.

Die Einziehung von Privatvermögen darf nur auf öffentliche Interessen gestützt werden, aufgrund eines Gesetzes ist eine gerechte Entschädigung zu leisten".

Dieser Verfassungsentwurf sieht im übrigen in Art. 44 vor, daß politische Parteien in öffentlichen Behörden keine Parteiabteilungen errichten dürfen, und anschließend heißt es:

"Art. 45: Politische Parteien müssen im gerechten Wettbewerb stehen und dürfen nicht staatliche Ressourcen ausschließlich oder überwiegend (allein) nutzen; außer der Herausgabe von Presseorganen dürfen sie weder in gewerbliche Unternehmen investieren noch gewerbliche Unternehmen betreiben.

124 Zhang Junhong, Taiwan gongshangren (Taiwanesische Unternehmer), S. 10.

125 Zhang Junhong, ebenda, S. 42.

126 Taiwan gongheguo xianfa caoan, abgedruckt in der Parteizeitung der Demokratischen Fortschrittspartei (DFP) Minjindang bao/Democratic Progressive News, Nr. 75 (1.9.1991) und in: Xiu xian shouce (Handbuch zur Verfassunsgreform), zusammengestellt von Gao Yongguang, Taipei 1991, S. 155 ff. 
Art. 46: Politische Parteien haben über die Herkunft ihrer Mittel Rechenschaft abzulegen.

Die Regierung hat die Parteien entsprechend ihrem in Wahlen erhaltenen Stimmenanteil finanziell zu unterstützen, die Art und Weise der Unterstützung wird durch Gesetz geregelt".

\section{Ausblick}

Die Verfassung der Republik China enthält ein Bekenntnis zugunsten des Privateigentums und der Wirtschaftsfreiheit. Modifikationen gibt es vor allen Dingen durch die auf dem politischen Konzept von Sun Yat-sen fußenden verfassungsrechtlichen Politikziele. Die Untersuchung hat ergeben, $\mathrm{da} ß$ die Untemehmerfreiheit in Taiwan allerdings erheblichen Einschränkungen unterworfen ist, nämlich Einschränkungen durch Sondergesetze, durch Staats- und durch Parteiunternehmen der KMT.

Der Bereich, in dem die Unternehmerfreiheit insbesondere verwirklicht worden ist, der Bereich der Exportwirtschaft, bildet auch den Bereich des taiwanesischen Wirtschaftserfolges. Die Forderungen aus Kreisen der Opposition, der Wirtschaft und der Wissenschaft nach einer umfassenden Liberalisierung der Wirtschaftsverfassung, d.h. vor allem die Abschaffung der Einschränkungen der Unternehmerfreiheit, welche vorrangig der Herrschaftssicherung der KMT-Elite dienen, erfahren durch diesen Wirtschaftserfolg ihre Berechtigung.

Wie bereits im Untertitel angedeutet, ist Taiwan nun an einem Wendepunkt angelangt. Mit der Beendigung der Periode der Mobilmachung zur Niederschlagung der Rebellion hat das Wirtschaftsministerium begonnen, Verordnungen mit Kriegsrechtscharakter abzuschaffen.127 Auch das Parlament arbeitet an der Abschaffung von restriktiven Sondergesetzen. Auf dem Gebiet der Abschaffung beziehungsweise gründlichen Uberarbeitung der Sondergesetze wird Taiwan bei seinem Wege zur Liberalisierung und Demokratisierung vermutlich noch am ehesten der Durchbruch gelingen.

Viel stärker griff die Regierung mit dem Aufbau von Staatsunternehmen in weiten Wirtschaftsbereichen des Binnenmarktes in die Unternehmerfreiheit ein. Nach mehrjähriger Diskussion innerhalb der Regierung ist im Juni 1991 diesbezüglich das "Gesetz über die Privatisierung öffentlicher Unternehmen" in geänderter Fassung verkündet worden.128 Und im Frühjahr 1992 hat das Wirtschaftsministerium einen konkreten Privatisierungsplan vor-

127 Zhongguo shibao vom 15.4.1991.

128 Gongying shiye yizhuan minying tiaoli vom 19.6.1991. 
gelegt. ${ }^{129}$ Seine Verwirklichung bleibt abzuwarten, betreffend die Staatsunternehmen sind jedenfalls erste Schritte hin zu einer Liberalisierung festzustellen. Ganz anders sieht es hingegen bei den Parteiunternehmen der KMT aus, hier ist zur Zeit sogar noch eine Ausweitung zu verzeichnen.

Die gegenwärtigen Reformbemühungen sind noch durch einen besonderen Faktor behindert, nämlich dem Gegensatz der taiwanesischen Mehrheit und der Minderheit der Chinesen vom Festland.130 Im öffentlichen Sektor, einschließlich der Staats- und Parteibetriebe nehmen die Festlandschinesen einen überproportionalen Teil ein, der mit steigender Verwaltungsstufe zunimmt. In der etwa dem deutschen höheren Dienst vergleichbaren Verwaltungslaufbahn bilden sie sogar einen Anteil von zur Zeit 57,8 \%. Ansonsten haben sie einen überproportional großen Anteil im Dienstleistungsgewerbe und selbst innerhalb der Großunternehmen stellten sie jahrelang einen über ihrem Bevölkerungsanteil hinausgehenden Anteil. Dieser somit nicht nur die Politik beherrschende sondern auch in der Wirtschaft festzustellende und einseitig zulasten der Taiwanesen gehende Unterschied zwischen beiden Bevölkerungsgruppen wird von den Chinesen vom Festland heruntergespielt oder bewußt geleugnet.131 Mit der schrittweisen, politischen Liberalisierung ist dieser seit Jahrzehnten angestaute Konflikt aufgebrochen und hat die KMT jetzt selbst voll erfaßt.

Um abschließend auf die eingangs gestellte Frage zurückzukommen ist zu sagen, daß man betreffend Taiwan von einer Übernahme der westeuropäischen Wirtschaftsordnung insgesamt - zumindest noch - nicht reden kann. Zu ihrer Herrschaftssicherung hat die regierende, vom chinesischen Festland stammende Flüchtlingselite der KMT die Unternehmerfreiheit insbesondere im Binnenmarkt marktfeindlichen Beschränkungen unterworfen. Im übrigen duldet sie eine sehr umfangreiche, illegale Schattenwirtschaft. Im ersten Falle liegt eine Hegemonie der Politik gegenüber der Wirtschaft vor und im zweiten Falle fehlt die erfor-

129 Siehe den Bericht in: The Free China Journal vom 10. April 1992.

130 Die Taiwanesen nehmen einen Anteil von $75 \%$, Festlandschinesen $14 \%$, Hakka $10 \%$. Siehe dazu die von den beiden Wissenschaftlem der Academia Sinica Lin Zhongzheng und Lin Heling vorgelegte Studie "Die wirtschaftlichen Unterschiede der verschiedenen ethnischen Gruppen in Taiwan" (Taiwan diqu ge zuqun de jingji chayi) in: Guojia zhengce yanjiu zhongxin/Institute for National Policy Research; Shengji, zuqun yu guojia rentong yantaohui lunwenji (Symposium über Provinzangehörigkeit, ethnische Gruppen und Bekenntnis zum Staat, Aufsatzsammlung) vom 12. April 1992.

131 Als ein typisches Beispiel mag hier Prof. Shaw Yu-ming, der Leiter des Presseamtes von 1987 bis 1991, herangezogen werden:"The relationship between these two groups in the early period was not an easy one, but this early uneasiness is almost gone today, mostly due to intermarriage and to more than three decades of living together in an island enviroment ... In the postwar period, there was the unfortunate incident of February 28, 1947, when the ruling mainland authorities clashed with some Taiwanese political activists, and this resulted in some bloodshed. Apart from that, the Taiwanese and the mainlanders have worked together for their mutual benefit", in: ders., Beyond the Economic Miracle, Taipei, 2. Aufl. 1989, S. 18 f. 
derliche Kontrolle der Politik gegenüber der Wirtschaft zum Schutze der Allgemeinheit, beides Abweichungen von einer westeuropäischen Wirtschaftsordnung. Allerdings - so ist noch einmal zu betonen - wird eine einer westeuropäischen Wirtschaftsordnung vergleichbare Liberalisierung seit langem in Taiwan gefordert und hat in den Teilbereichen, wo sie umgesetzt worden ist, auch zu dem taiwanesischen Wirtschaftsaufschwung beigetragen. 
is also discussed. The report ends with a number of conclusions of mainly legal character and some recommendations the delegation has addressed to the Austrian government, which, however, may also be of general relevance.

\section{The Economic Constitution of Taiwan - The Situation at the Turning Point}

\section{By Wolfgang Lasars}

Taiwan, that is the Republic of China, understands herself as the anti-communist representative of a free China, on the other hand liberals in Taiwan demand a fundamental marketoriented reform of the Economic Constitution. To understand this contradiction the article examines the Economic Constitution exemplified by the development and the extent of the freedom of enterprises in Taiwan.

On one hand the system of private property and the freedom of enterprises are guaranteed by the Constitution. On the other hand emergency laws which go back to World War II and the civil war against the Chinese Communists have restricted the freedom of enterprises for several decades until 1992. The freedom of enterprises is even more curbed by a large sector of public enterprises and a special sector of corporations owned directly by the ruling party KMT. Because of the dominance of the privileged corporations of the state and the party KMT in the domestic market, many small and medium sized companies have to turn to the export sector. Another related aspect of the Economic Constitution of Taiwan is a large area of illegal but tolerated economic activity. From the beginning onwards, the liberal and democratic opposition in Taiwan has stringly criticized this Economic constitution imposed by the KMT.

Now Taiwan has reached a tuming point: Most probably, the emergency laws will be scrapped, concerning the public corporations the government has initialled first steps in the direction of privatization. However, on the other hand, the corporate sector of the ruling party KMT is still growing. It remains to be seen to which results the present reform process of the Economic Constitution finally will lead.

Mongolia on the Way to Pluralistic Democracy and Market Economy - The New Mongolian Constitution of 1992

\section{By Jürgen Merz}

The communist breakdown in the USSR lead to significant political and constitutional changes in Mongolia as well and initiated a period of transition from totalitarianism and 